\title{
Defining trained immunity and its role in health and disease
}

\begin{abstract}
Mihai G. Netea $\left(^{1,2,3 凶}\right.$, Jorge Domínguez-Andrés $^{1,2}$, Luis B. Barreiro ${ }^{4,5,6}$, Triantafyllos Chavakis ${ }^{7,8}$, Maziar Divangahi, 10,11, Elaine Fuchs ${ }^{12}$, Leo A. B. Joosten ${ }^{1,2}$, Jos W. M. van der Meer ${ }^{1,2}$, Musa M. Mhlanga ${ }^{13,14}$, Willem J. M. Mulder15,16,17, Niels P. Riksen ${ }^{1,2}$, Andreas Schlitzer ${ }^{18}$, Joachim L. Schultze ${ }^{3}$, Christine Stabell Benn ${ }^{19}$, Joseph C. Sun 20,21,22, Ramnik J. Xavier ${ }^{23,24}$ and Eicke Latz ${ }^{25,26,27 凶}$
\end{abstract}

Abstract | Immune memory is a defining feature of the acquired immune system, but activation of the innate immune system can also result in enhanced responsiveness to subsequent triggers. This process has been termed 'trained immunity', a de facto innate immune memory. Research in the past decade has pointed to the broad benefits of trained immunity for host defence but has also suggested potentially detrimental outcomes in immune-mediated and chronic inflammatory diseases. Here we define 'trained immunity' as a biological process and discuss the innate stimuli and the epigenetic and metabolic reprogramming events that shape the induction of trained immunity.

\section{Pattern recognition receptors (PRRs). Germline-encoded receptors that recognize pathogen-associated molecular patterns - evolutionarily conserved structures associated with pathogens such as viruses, bacteria, fungi and parasites - and damage-associated molecular patterns, which are exposed in damaged host tissues. There are four main families of PRRs: namely, Toll-like receptors, NOD-like receptors, C-type lectin receptors and RIG 1 -like receptors. Interaction of pathogen-associated molecular patterns with pattern recognition receptors mediates recognition of pathogens and triggers inflammation.}

\footnotetext{
凶e-mail: Mihai.Netea@ radboudumc.nl; eicke.latz@ uni-bonn.de

The vertebrate immune system has traditionally been divided into innate and adaptive arms. Cells of the innate immune system recognize pathogens and tissue damage through germline-encoded pattern recognition receptors (PRRs) $)^{1,2}$, which sense diverse pathogen-associated molecular patterns and damage-associated molecular patterns. The processes activated on engagement of PRRs are rapid, are considered to be non-specific and include responses such as phagocytosis, cell locomotion, killing of pathogens or cells, and cytokine production. These innate immune mechanisms are usually very effective in eliminating invading pathogens. Additionally, dendritic cells (DCs) and specialized T cells and B cells drive adaptive immune responses, which can be concomitantly induced. These lymphocyte-dependent adaptive immune responses are slower to develop but are antigen specific and lead to long-term immunological memory ${ }^{3}$.

For a long time it was assumed that immunological memory was an exclusive hallmark of the adaptive immune response. However, a growing body of literature indicating that innate immune cells - and even tissue-resident stem cells - can show adaptive characteristics has challenged this $\operatorname{dogma} \mathrm{g}^{4-8}$. Greater protection against reinfection - a de facto immune memory function - has also been reported in plants and invertebrates $^{9-11}$, which lack an adaptive immune system. This demonstrates that adaptation of host defence can occur on the basis of innate-like immune mechanisms. Moreover, certain infections and vaccinations can induce broad protection against other pathogens through innate immune mechanisms $\mathrm{s}^{5,12}$. Conversely, the phenomenon called 'LPS tolerance', which can be induced by low doses of lipopolysaccharide (LPS) and other Toll-like receptor ligands, is also an adaptation of cellular responses to an external stimulus, but which leads to a lower inflammatory response to a second stimulation ${ }^{13}$.

These studies have led to the hypothesis that the innate immune system also exhibits adaptive characteristics, a property that has been termed 'trained immunity'. Understanding the properties of trained immunity will result in a better understanding of host defence mechanisms and the pathogenesis of immune-mediated diseases. The conceptual and mechanistic advances in this emerging field of science will open new avenues for clinical applications in vaccination as well as for disease prevention and treatment. In this Review, we discuss the latest discoveries in the field of trained immunity and highlight possible directions of future research in this field.

\section{Defining trained immunity}

The concept of trained immunity describes the long-term functional reprogramming of innate immune cells, which is evoked by exogenous or endogenous insults and which leads to an altered response towards a second challenge after the return to a non-activated state. The secondary response to the subsequent non-specific stimulus can be altered in such a way that the cells respond more or less strongly than to the primary response, conferring context-adjusted and time-adjusted responses. It is important to underline that trained immunity represents the concept of long-term adaptation of innate 
Bacillus Calmette-Guērin (BCG). An attenuated form of the bacterium Mycobacterium bovis, which is the causative agent of bovine tuberculosis Developed at Institut Pasteur twentieth century as a vaccine to prevent tuberculosis (BCG vaccine), it also induces protective heterologous effects against infections and at the beginning of the malignancies.

immune cells rather than a particular transcriptional or functional programme: indeed, different stimuli (for example, $\beta$-glucan, LPS or the bacillus Calmette-Guerin (BCG) vaccine) can induce different trained immunity programmes.

In contrast to adaptive immune responses, epigenetic reprogramming of transcriptional pathways - rather than gene recombination - mediates trained immunity (FIG. 1). The immunological phenotype of trained immunity has been proven to last at least 3 months and up to 1 year, although heterologous protection against infections induced by live vaccines can last for up to 5 years ${ }^{14}$. However, even considering this, trained immunity is generally reversible and shorter lived than classical epitope-specific adaptive immunological memory ${ }^{12,15}$. Importantly however, recent studies have suggested

\footnotetext{
Author addresses

${ }^{1}$ Department of Internal Medicine and Radboud Center for Infectious Diseases, Radboud University Medical Center, Nijmegen, Netherlands.

${ }^{2}$ Radboud Institute for Molecular Life Sciences, Radboud University Medical Center, Nijmegen, Netherlands.

${ }^{3}$ Department of Genomics and Immunoregulation, Life and Medical Sciences Institute, University of Bonn, Bonn, Germany.

${ }^{4}$ Department of Genetics, CHU Sainte-Justine Research Centre, Montreal, QC, Canada. ${ }^{5}$ Department of Pediatrics, University of Montreal, Montreal, QC, Canada.

${ }^{6}$ Genetics Section, Department of Medicine, The University of Chicago, Chicago, IL, USA. 'Institute for Clinical Chemistry and Laboratory Medicine, University Hospital and Faculty of Medicine Carl Gustav Carus of TU Dresden, Dresden, Germany.

${ }^{8}$ Centre for Cardiovascular Science, Queen's Medical Research Institute, University of Edinburgh, Edinburgh, UK.

${ }^{9}$ Meakins-Christie Laboratories, Department of Medicine, McGill University Health Centre, Montreal, QC, Canada.

${ }^{10}$ Department of Microbiology and Immunology, McGill University, Montreal, QC, Canada. ${ }^{11}$ McGill International TB Centre, McGill University Health Centre, Montreal, QC, Canada.

${ }^{12}$ Howard Hughes Medical Institute, Robin Chemers Laboratory of Mammalian Cell

Biology and Development, The Rockefeller University, New York, NY, USA.

${ }^{13}$ Division of Chemical and Systems Biology, Department of Integrative Biomedical

Sciences, Faculty of Health Sciences, Institute of Infectious Disease and Molecular

Medicine, University of Cape Town, Cape Town, South Africa.

${ }^{14}$ Gene Expression and Biophysics Unit, Instituto de Medicina Molecular, Faculdade de Medicina Universidade de Lisboa, Lisbon, Portugal.

${ }^{15}$ Translational and Molecular Imaging Institute, Department of Radiology, Icahn School of Medicine at Mount Sinai, New York, NY, USA.

${ }^{16}$ Department of Oncological Sciences, Icahn School of Medicine at Mount Sinai, New York, NY, USA

${ }^{17}$ Laboratory of Chemical Biology, Department of Biomedical Engineering and Institute for Complex Molecular Systems, Eindhoven University of Technology, Eindhoven, Netherlands.

${ }^{18}$ Myeloid Cell Biology, Life and Medical Sciences Institute, University of Bonn, Bonn, Germany.

${ }^{19}$ Bandim Health Project, OPEN, Department of Clinical Research, University of Southern Denmark, Odense, Denmark.

${ }^{20} \mathrm{Immunology}$ Program, Memorial Sloan Kettering Cancer Center, New York, NY, USA.

${ }^{21}$ Louis V. Gerstner Jr. Graduate School of Biomedical Sciences, Memorial Sloan Kettering Cancer Center, New York, NY, USA.

${ }^{22}$ Department of Immunology and Microbial Pathogenesis, Weill Cornell Medical College, New York, NY, USA.

${ }^{23}$ Broad Institute of MIT and Harvard, Cambridge, MA, USA.

${ }^{24}$ Center for Computational and Integrative Biology, Massachusetts General Hospital and Harvard Medical School, Boston, MA, USA.

${ }^{25}$ Institute of Innate Immunity, University Hospital, University of Bonn, Bonn, Germany.

${ }^{26}$ Division of Infectious Diseases and Immunology, University of Massachusetts Medical School, Worcester, MA, USA.

${ }^{27}$ German Center for Neurodegenerative Diseases, Bonn, Germany.
}

transgenerational effects through induction of trained immunity ${ }^{16,17}$.

\section{Immune memory: an evolutionary perspective}

The adaptive immune system, in which T cells and B cells mediate immunological memory, has developed relatively recently in vertebrates (that is, around 500 million years ago). By contrast, invertebrate species rely solely on the innate immune system for defence against pathogens and recognition of tissue damage. In vertebrates, on encounter with a threat and activation of particular lymphocyte clones that recognize specific antigens from the invading pathogens, de novo rearrangement of immunoglobulin and $\mathrm{T}$ cell receptor gene segments occurs. This 'on demand' production of novel and diverse receptors forms the basis for lymphocyte-mediated specific immune memory responses in jawed vertebrates (the gnathostomes $)^{18}$. An alternative adaptive immune system evolved in jawless vertebrates, in which specific lymphocyte-mediated immune memory is mediated by variable lymphocyte receptors generated via the somatic rearrangement of gene elements encoding leucine-rich repeat motifs ${ }^{19}$. Both immunoglobulin-based and variable lymphocyte receptor-based adaptive immune processes in highly differentiated immune cells (that is, memory $\mathrm{T}$ cells and B cells or gnathostome lymphocytes) induce a recall immune response to the previously encountered pathogen that is both stronger and highly specific to the pathogen, ensuring improved survival of the organism.

Given the evolutionary success of organisms lacking adaptive immune responses, which represent up to $97 \%$ of the total biodiversity on Earth ${ }^{20}$, it is unlikely that immunological memory has evolved only in vertebrates. Indeed, over the past two decades, an increasing number of studies have provided evidence that the immune system of plants and invertebrates may be 'primed' by an initial infection, leading to protection against subsequent infections s $^{10,11,18,19,21,22}$. Likewise, memory characteristics in the innate immune system of vertebrates have recently been described and referred to as 'trained immunity', a process that results in a heightened reaction to secondary infections or sterile triggers of inflammation ${ }^{5,12}$. Although trained immunity is controlled by distinctive mechanisms and is less specific and of shorter duration than adaptive immune memory ${ }^{23}$, both fulfil the same principal function: a quicker and stronger response against pathogens and improved survival of the host.

\section{Trained immunity in vertebrates}

Evidence of trained immunity in mouse infection models. Many studies in mice have documented the existence of adaptive characteristics of innate immunity. Together, these studies demonstrated that training mice with different microbial ligands could protect against subsequent lethal infection in a non-specific manner. For example, treatment with the fungal ligand $\beta$-glucan protected against subsequent infection with Staphylococcus aureus $^{24,25}$, while the peptidoglycan component muramyl dipeptide induced protection against Streptococcus pneumoniae and Toxoplasma gondii infections ${ }^{26}$. Other examples include $\mathrm{CpG}$ oligodeoxynucleotide application, 


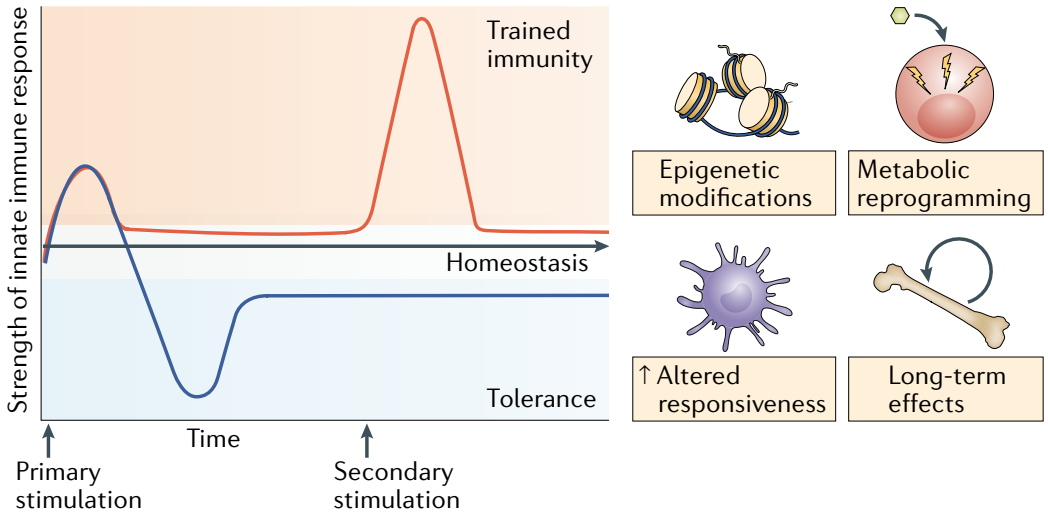

Fig. 1 | Trained immunity and tolerance: two opposite functional programmes of innate immunity. Infections or sterile tissue triggers induce inflammation and the activation of immune effector mechanisms. Concomitant to a proinflammatory response, anti-inflammatory mechanisms are provoked to prevent overshooting inflammation and tissue damage and to limit the inflammatory response in time. Trained immunity involves epigenetic and metabolic reprogramming of the innate immune cells, allowing qualitatively and quantitatively adjusted responses of innate immune cells to subsequent time-delayed heterologous stimulation. Misguided trained immunity responses can contribute to disease progression, resulting in either a chronic hyperinflammatory state or a persistent state of immunological tolerance, a mechanism that dampens the inflammatory response of the host to maintain homeostasis and prevent tissue damage and organ failure, with the subsequent risk of secondary infections and other diseases related to decreased activity of the immune system.

\section{Myeloid cells \\ Cells of the immune system that arise from pluripotent primordial cells in the bone marrow. Myeloid cells (monocytes, macrophages, dendritic cells and \\ granulocytes) have many physiological roles, among which are roles are to destroy the invading pathogens and repair tissues.}

Chromatin

A complex structure composed of DNA and proteins located in the nucleus in which the genetic material of eukaryotic cells is organized. Chromatin has a high degree of organization, which allows the compaction of the genetic material, but this remains reachable to allow access of the protein machinery that regulates gene transcription. Chemical modification of histones, the core proteins in chromatin, regulates the accessibility of the DNA for the transcription machinery. which leads to protection against subsequent experimental sepsis and Escherichia coli meningitis ${ }^{27}$, or flagellin-induced protection against S. pneumoniae ${ }^{28}$ and rotavirus ${ }^{29}$.

These data suggest that infections by themselves or the exposure of the immune system to microorganismderived immune stimulatory agents can provoke not only specific protection against reinfection but also non-specific protection against a subsequent challenge with the same or another pathogen. For example, vaccination with the BCG vaccine was shown to protect animals against secondary infections with Candida albicans, Schistosoma mansoni and Mycobacterium tuberculosis ${ }^{30-32}$. The non-specific character of the protection argues against a significant role of adaptive immunity for mediating this type of cross protection and suggests activation of rather non-specific protective innate immune mechanisms. The BCG vaccine also protects against lethal candidiasis in SCID mice, which lack functional $\mathrm{T}$ cells and B cells ${ }^{23,33,34}$. Similarly, a prior mild infection with $C$. albicans protects against the subsequent exposure to a normally lethal candidiasis infection ${ }^{35}$ in both athymic mice and in recombination-activating gene 1 (RAG1)-deficient mice, further demonstrating the $\mathrm{T}$ cell-independent mechanisms of trained immunity ${ }^{36,37}$. In these studies, the ability of a prior infection to provide protection against infection with unrelated pathogens was dependent on macrophages ${ }^{35}$ and on proinflammatory cytokine production ${ }^{38}$. Importantly, a recent study showed that multiple passages of C. albicans through the gut of mice, leading to an adaptation of the fungus towards colonization, results in a stronger capacity to induce trained immunity and improved protection against non-specific infections in a lymphocyte-independent manner ${ }^{39}$.
Trained immunity in humans. An increasing body of evidence suggests that trained immunity plays a critical role in humans. First, an extensive collection of epidemiological data argues that live vaccines such as the BCG vaccine, measles vaccine, smallpox vaccine and oral polio vaccine have beneficial, non-specific protective effects against infections other than the target diseases ${ }^{40-47}$ (for a review, see also $\mathrm{REF}^{48}$ ). Subsequently, proof-of-principle trials with the BCG vaccine in adults ${ }^{23,49}$ and children ${ }^{50,51}$ demonstrated that this vaccine induces non-specific activation of innate immune cells. Interestingly, both epidemiological and immunological studies have shown that the vaccine effects may last for months, but may also be modified or even reversed when a non-live vaccine is given ${ }^{52,53}$. Furthermore, BCG vaccination led to protection against microorganisms in models of controlled human infection, such as yellow fever ${ }^{54}$ or malaria ${ }^{55}$, and this was associated with an augmented proinflammatory activity of monocytes (BOX 1). Second, certain infections, such as malaria, induce a state of hyper-responsiveness that is functionally equivalent to induction of trained immunity ${ }^{56-58}$. Finally, there is evidence that BCG vaccination can induce antitumour immune effects leading to the prevention or treatment of malignancies such as bladder cancer ${ }^{59}$, melanoma ${ }^{60}$, leukaemia ${ }^{61}$ and lymphoma ${ }^{62}$. Notably, these anticancer effects of BCG seem to be dependent on its capacity to induce trained immunity in monocytes and macrophages ${ }^{63}$.

Diversity of cells that can develop trained immunity. The cellular basis of the protection induced by trained immunity during bacterial and fungal infections resides in the functional reprogramming of myeloid cells. Some of the first evidence that macrophages have adaptive features came from investigations of LPS tolerance, which demonstrated that gene-specific chromatin modifications were associated with the silencing of genes coding for inflammatory molecules and the priming of other genes encoding antimicrobial molecules ${ }^{64}$. Subsequent studies showed that exposure of monocytes or macrophages to C. albicans or the fungal cell wall component $\beta$-glucan enhanced the subsequent response of these myeloid cells to stimulation with unrelated pathogens or pathogenassociated molecular patterns $\mathrm{s}^{37}$. Induction of trained immunity in monocytes was accompanied by the alteration of several chromatin marks ${ }^{37,65}$. In addition to the data from infections with bacterial and fungal pathogens, other studies have shown that monocytes or macrophages can mount trained immune responses following infection with parasites ${ }^{66}$. Recent work has shown that DCs can also show immune memory responses. In this regard, DCs isolated from mice exposed to the fungal pathogen Cryptococcus neoformans displayed strong interferon- $\gamma$ (IFN $\gamma)$ production and enhanced proinflammatory cytokine responses on subsequent challenge, which is indicative of a memory response ${ }^{67}$. These effects were dependent on epigenetic changes and were impaired by the treatment of mice with a histone methyltransferase inhibitor ${ }^{67}$.

Certain viral infections also exert protective effects independently of adaptive immunity. For example, herpesvirus latency increases resistance to the bacterial 


\section{Box 1 | Trained immunity as a therapeutic target}

Regulating trained immunity can be a powerful therapeutic paradigm in different disease contexts ${ }^{176}$. Depending on the condition, it could be beneficial to induce trained immunity to aid specific cancer therapies or to treat the immune paralysis associated with sepsis. Other exciting therapeutic applications would be to inhibit an overly trained innate immune state in chronic inflammatory diseases or to prevent potential detrimental trained immunity in organ transplantation.

Promoting trained immunity is particularly relevant for the prevention of child death and morbidity; for instance, providing bacillus Calmette-Guérin (BCG) to low-weight African children at birth rather than in the subsequent weeks or months is associated with a reduction of neonatal mortality by a third ${ }^{40}$. Also, it could be very relevant in the treatment of cancer and can be achieved by activating certain pattern recognition receptors. Indeed, the first immunotherapeutic strategy in cancer, developed by William Coley ${ }^{177}$ at the end of the nineteenth century, induced inflammation and may have been linked to induction of a trained immunity phenotype. After observing spontaneous tumour remission in patients with cancer with concomitant infections, Coley developed a method involving injection of streptococcal organisms into tumours ${ }^{178}$. Despite successful treatment of several patients, Coley's approach was met with criticism and scepticism because of its unpredictability and the risk of promoting dangerous systemic inflammation. Approximately half a century later, in the late 1950s, the BCG vaccine was developed into a novel immunotherapy to treat cancer by Lloyd Old and colleagues ${ }^{179}$. Currently, BCG vaccination is a US Food and Drug Administration-approved treatment modality for bladder cancer, and although not currently part of standard clinical care, it is also used for other treatment of malignancies such as lymphoma and melanoma. $\beta$-Glucan has long been used in East Asia to boost immune responses in patients with cancer, and it is currently in clinical trials in the USA in combination with checkpoint inhibitors ${ }^{180-182}$. Several alternative strategies to activate innate immunity and thereby trained immunity by injecting immunostimulatory agents into tumours are currently being developed together with checkpoint blockade, including activators of the NLRP3, STING, Toll-like receptor and RIG1 pathways. Trained immunity-induced rebalancing of macrophage and DC function may overcome the immunosuppressive microenvironment, facilitating the performance of existing $T$ cell immunotherapies, chimeric antigen receptor T cell therapy ${ }^{183}$ and checkpoint inhibitors ${ }^{184}$. The challenge in the coming years will be to identify potent agents that induce antitumoural trained immunity processes without promoting excessive systemic side effects. Given that the host microbiota determines the effectiveness of checkpoint blockade therapy in cancers ${ }^{185}$, it is of particular relevance to mechanistically untangle the effect of the host microbiota on the effectiveness of trained immunity. macrophages, yet occur in the absence of RAG-mediated antigen receptor gene rearrangement ${ }^{82}$, this unique NK cell response may represent an evolutionary bridge between the memory response of $\mathrm{T}$ cells and that of myeloid lineage cells ${ }^{78,83}$.

A response in innate-like lymphocytes that more closely resembles trained immunity is perhaps the non-antigen specific priming of NK cells and innate lymphoid cells (ILCs) by proinflammatory cytokines. Mouse and human NK cells exposed to IL-12 and IL-18 showed more robust production of IFN $\gamma$ weeks after initial priming ${ }^{84,85}$, and transplantation of these cytokine-induced memory-like NK cells has shown efficacy in clinical trials to treat leukaemias ${ }^{86}$. Even in the adaptive responses of MCMV-specific NK cells, proinflammatory cytokine signals are critical for the formation of robust effector and memory NK cells ${ }^{87-89}$. It was recently found that liver-resident group 1 ILCs (ILC1s) also expand and persist after infection with MCMV, acquiring stable transcriptional, epigenetic and phenotypical changes 1 month after the resolution of systemic infection, and showed enhanced memory responses ${ }^{90}$. In this setting, both proinflammatory cytokines and antigen specificity (NK1.1 receptor-mediated recognition of MCMV-encoded m12) were critical in driving a memory response in ILC1 $s^{90}$. ILC2s have also been suggested to possess features of immunological memory following stimulation with allergens ${ }^{91}$, perhaps resembling prior studies of hapten-induced priming of NK cells in delayed hypersensitivity responses ${ }^{92}$. Anamnestic immunity in NK cells may also play an important role in physiological processes such as pregnancy. A recent study showed that NK cells from women who have been repeatedly pregnant show a particular epigenetic and transcriptomic landscape, with open chromatin around the enhancers of IFNG and VEGFA, improving placentation and supporting vascular sprouting ${ }^{93}$.

pathogens Listeria monocytogenes and Yersinia pestis ${ }^{68}$, with protection achieved through enhanced production of IFN $\gamma$ and systemic activation of macrophages. In the past decade, a unique anamnestic response has been described in natural killer (NK) cells during cytomegalovirus infection. Experimental studies demonstrated that mouse and human NK cells possess adaptive immune characteristics following infection with mouse cytomegalovirus (MCMV) or human cytomegalovirus, respectively ${ }^{69-73}$. Mouse NK cells bearing the Ly49H receptor possess antigen specificity for MCMV-encoded glycoprotein $\mathrm{m} 157\left(\mathrm{REFS}^{74,75}\right)$, can undergo clonal proliferation (as much as $10^{4}$-fold expansion from a single NK cell clone $)^{76,77}$ and persist during the contraction and memory phases similarly to $\mathrm{CD}^{+} \mathrm{T}^{+}$cells ${ }^{78}$. On reinfection, these memory NK cells undergo a secondary expansion and can more rapidly degranulate and release cytokines, resulting in a more protective immune response against $\mathrm{MCMV}^{69,79}$. In human NK cells, the NKG2C receptor can mediate a similar function through recognition of human cytomegalovirus-encoded UL40 peptides presented on the non-classical MHC molecule HLA- ${ }^{80,81}$. Because these adaptive NK cell responses more closely resemble $\mathrm{T}$ cell responses than trained immunity in

\section{Trained immunity in stromal and epidermal stem cells.}

Intriguingly, the induction of innate immune memory is not exclusively confined to immune cells but can also occur in stromal and epithelial cells. The discovery of inflammatory memory behaviour in epidermal stem cells is of particular relevance, as tissue stem cells are the cornerstone of regeneration in homeostasis and they reside in distinct microenvironments or niches and exchange signals that define their tasks and their molecular behaviours ${ }^{6,94}$. Although the rate of cellular replacement during homeostasis is tissue and context specific, even quiescent stem cells are mobilized into action on tissue injury. Similarly, inflammation and infections perturb the niche microenvironment, which can override the normal homeostatic cues and prompt changes in stem cell behaviours. Thus, through their ability to sense and respond to niche signals, stem cells can adjust to and survive stressful situations. Remarkably, stem cells form an enduring epigenetic memory from such encounters, which equips them with the ability to mobilize more rapidly during subsequent assaults ${ }^{6,95}$.

Stem cells express receptors for several inflammatory mediators, which enables them to adjust to their specific inflammatory milieu ${ }^{96-98}$. Moreover, stem cells 
are equipped with receptors to sense whether the epithelial barrier is breached and, in turn, actively recruit immune cells to prevent spread of bacteria and to repair the damage ${ }^{7}$. Although it is still unfolding, the molecular communication avenue between stem cells and immune cells appears to be bidirectional. Stem cells do not merely take instructions but rather they also actively instruct the immune system. In the skin, for example, stem cells can sense when their niche barrier is breached and produce signals to recruit specific immune cell sentinels, even under conditions where the immune system itself has been suppressed ${ }^{7}$. In turn, recruited immune cells can signal to stem cells to proliferate and patch the barrier. In this way, the coordination is honed to achieve maximal tissue repair. Another cell type that can acquire a trained immunity-like phenotype is the fibroblast. It was demonstrated that IFN $\beta$ treatment of mouse embryonic fibroblasts led to faster and higher induction of interferon-stimulated genes that

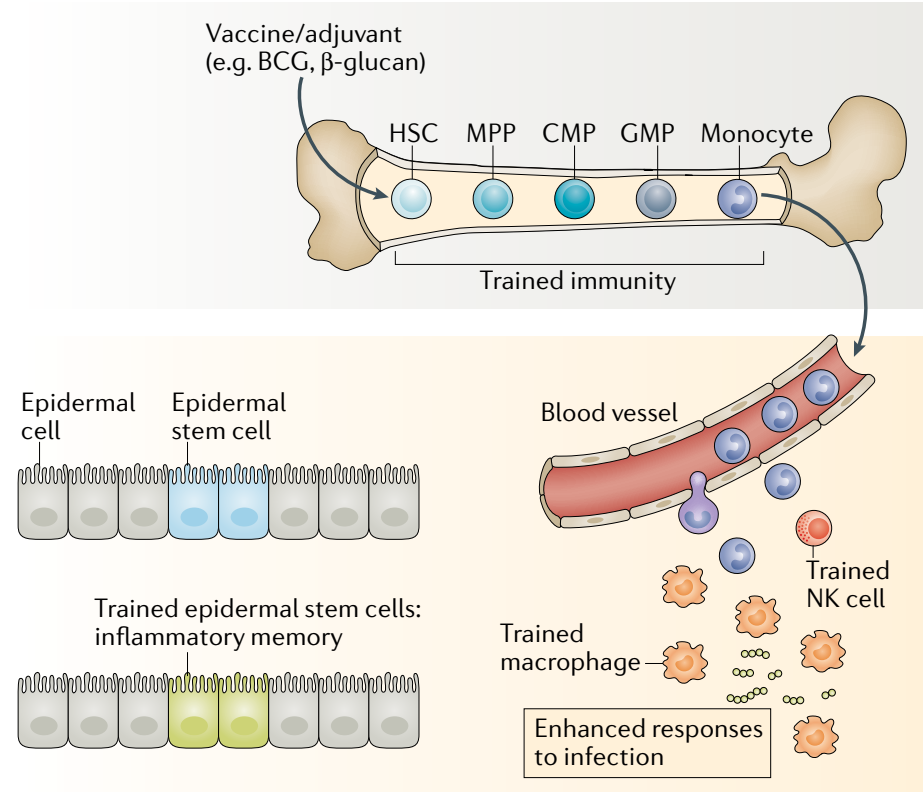

Central trained immunity

Peripheral trained immunity

Fig. 2 | Central and peripheral trained immunity. Although trained immunity was first established in cells of the mononuclear phagocyte lineage (that is, monocytes and macrophages), monocytes have a relatively short lifespan and are unlikely to transmit their memory phenotype to their progeny and provide sustainable protection. Thus, current vaccine strategies that directly target monocytes or macrophages may have limited capacity for generating sustained innate immune memory. By contrast, haematopoietic stem cells (HSCs) are long-lived cells with self-renewal properties that reside in the bone marrow. The bone marrow is the site of haematopoiesis where HSCs continually undergo asymmetric division giving rise to the full repertoire of myeloid and lymphoid cell types. HSCs can directly respond to acute and chronic infections. Although the exact mechanisms of precursor proliferation or differentiation are not well understood, persistent activation of HSCs can result in their exhaustion, leading to devastating effects on the systemic immune compartment. Monocytes derived from trained HSCs migrate to peripheral organs, where they give rise to monocyte-derived macrophages with enhanced effector functions against different types of pathogens. Natural killer (NK) cells possess adaptive immune characteristics following infection. On reinfection, these memory NK cells undergo a secondary expansion and can more rapidly degranulate and release cytokines, resulting in a more protective immune response. Epithelial stem cells show memory functions during human allergic inflammatory disease, displaying changes in the chromatin accessibility when the stimulus is withdrawn. BCG, bacillus Calmette-Guérin; CMP, common myeloid progenitor: GMP, granulocyte-macrophage progenitor; MPP, multipotent progenitor. correlated with enhanced recruitment of polymerase II to interferon-stimulated gene loci on restimulation ${ }^{99}$.

\section{Central versus peripheral trained immunity}

Trained immunity was initially shown to act through mature myeloid cells. Until recently this hypothesis resulted in a conundrum as mature myeloid cells, such as monocytes and DCs, in both mice and humans are short-lived, with an average half-life of 5-7 days ${ }^{100-102}$. Therefore, how trained immunity can be maintained in myeloid cells for several months, years and even decades ${ }^{41}$ remained unknown. More recent work has helped to resolve this issue by showing that trained immunity can occur in bone marrow progenitor cells (central trained immunity), as well as in blood monocytes and tissue macrophages (peripheral trained immunity) (FIG. 2).

Recent studies have shown that $\beta$-glucan or BCG can reprogramme myeloid progenitors in the bone marrow to generate trained immunity within the myeloid cell compartment ${ }^{103}$. In a mouse model of tuberculosis, Kaufmann and colleagues ${ }^{32}$ demonstrated that BCG vaccination reprogrammes haematopoietic stem cells (HSCs) in the bone marrow towards myelopoiesis in an IFN $\gamma$-dependent manner, which leads to protective trained immunity. Similarly, $\beta$-glucan increases myelopoiesis by promoting the expansion of myeloid-biased CD $41^{+}$HSCs and cells from the myeloid-biased multipotent progenitor 3 (MPP3) subset ${ }^{104}$. IL-1 $\beta$ and granulocyte-macrophage colony-stimulating factor (GM-CSF) signalling as well as alterations in glycolysis and cholesterol biosynthesis in bone marrow progenitors are putative mechanisms that have been proposed to explain $\beta$-glucan-induced trained immunity in mice ${ }^{104}$.

The discovery that HSCs, similarly to epithelial stem cells, display a memory function could explain the long-standing mystery as to why short-lived immune cells such as monocytes can acquire memory. Indeed, respiratory epithelial progenitors become more stem-like during human allergic inflammatory disease, and the associated accessible chromatin changes differ in their ability to return to normal when the stimulus is withdrawn ${ }^{105}$.

Several studies have furthermore investigated whether trained immunity exists at the level of individual tissues and if so, how these changes are maintained or erased to ensure proper tissue function. Conceivably, tissues exposed to the outside world, such as the skin, the lungs and the intestine, are prone to encounter immune training-inducing stimuli. This concept was explored in the lung using two models of viral challenge: namely, latent gammaherpesvirus infection and adenovirus infection $^{106,107}$. The severity of house dust mite-induced asthma was decreased in the lungs of mice that had previously been chronically infected with gammaherpesvirus $^{106}$. This phenotype was dependent on the long-term generation and maintenance of monocyte-derived regulatory alveolar macrophages that conferred protection against the development of an allergic response in the lung. Conversely, adenovirus infection induced remodelling in alveolar macrophages, which are long-lived tissue-resident cells, such that they retained the information of an inflammatory history and subsequently 
induced more pronounced antibacterial immunity ${ }^{107}$. However, in the case of the adenovirus infection, the tissue-specific training phenotype was dependent on the polarization of $\mathrm{CD}^{+} \mathrm{T}$ cells by trained alveolar macrophages and therefore provided a link between trained and adaptive immunity. Together, these two recent studies highlight the importance of tissue-specific training cues, which are likely to occur consecutively over an individual's lifespan, raising the question of how these training cues contribute to protection against infections and the development of inflammatory diseases and cancer.

\section{Epigenetic reprogramming}

Induction of a trained immune phenotype in innate immune cells enables them to react with stronger, more rapid or qualitatively different transcriptional responses when challenged with subsequent triggers. The molecular basis of this altered responsiveness of a defined subset of inflammatory genes is only partially understood, but evidence supports the convergence of multiple regulatory layers, including changes in chromatin organization at the level of the topologically associated domains (TADs), transcription of long non-coding RNAs (lncRNAs), DNA methylation and reprogramming of cellular metabolism (FIG. 3).

In quiescent myeloid cells, most of the proinflammatory gene loci are in a repressed configuration ${ }^{108}$, hindering access of the transcriptional machinery to the regulatory regions driving expression of inflammatory factors $^{109}$. Many studies have demonstrated that stimulation of innate immune cells can leave an 'epigenetic scar' at the level of stimulated genes, changing the long-term responsiveness of the cells that manifests itself as functional trained immunity programmes. Intrinsic to the presence of such an epigenetic scar is the question of how it is selectively directed to specific locations in the genome, either at the promoters of stimulated genes or at distal regulatory elements. Two key epigenetic marks accompany trained immunity: the acquisition of histone 3 lysine 27 acetylation (H3K27ac) marks at distal enhancers (marked with histone 3 lysine 4 methylation (H3K4me1)) and the consolidation of histone 3 lysine 4 trimethylation ( $\mathrm{H} 3 \mathrm{~K} 4 \mathrm{me} 3)$ marks at the promoters of stimulated genes (FIG. 4). Transcription is an inherently stochastic process, which is regulated by several factors, including the random collision of transcription factors with upstream promoter and enhancer DNA regions. However, dynamic loop-mediated regulation and the resulting stochastic responses in gene expression may not be ideal for gene classes that need to respond both immediately and uniformly to external stimuli across cell populations. This suggests that to reduce stochasticity in gene expression, the chromosomal contact between rapidly responding genes may exist in a more stable or preformed state, which strongly implicates the influence of chromosomal looping and TAD structure on this subclass of transcriptional responses. Studies using Hi-C (a method for analysing chromatin interactions) have revealed that several classes of innate immune genes are segregated into TADs and interact within multigene complexes. A recent study demonstrated how TAD structure enables a class of lncRNAs called 'immune gene-priming lncRNAs' (IPLs) to be brought into close proximity with transcriptionally poised innate immune genes, before their transcriptional activation $^{110}$. IPLs exploit preformed 3D looping contacts to bring the $\mathrm{H} 3 \mathrm{~K} 4 \mathrm{me} 3$ histone-modifying complex close to the promoters of highly responsive innate immune genes, permitting their epigenetic activation and training. The insertion of an IPL in the mouse preformed chemokine TAD confers these genes with the ability to transcriptionally respond rapidly and robustly (uniformly across all cells in the population) and to be accessible to training by $\beta$-glucan. The latter is a property they previously did not have in the absence of the IPL ${ }^{110}$. As current studies have investigated the role of IPLs only in $\beta$-glucan-induced trained immunity phenotypes, further studies are warranted to explore these mechanisms in other experimental settings, including the importance of IPLs during in vivo vaccination.

This functional experiment demonstrates the key role IPLs play in the 'writing' of $\mathrm{H} 3 \mathrm{~K} 4 \mathrm{me} 3$ marks at discrete loci in the genome ${ }^{110}$. How the H3K27ac mark is directed to specific distal enhancer marks (carrying H3K4me1 epigenetic marks) remains an important outstanding question. It is clear from mouse HSC data that after BCG exposure, the acquisition of open chromatin as measured by assay for transposase-accessible chromatin using sequencing (ATAC-seq) begins in HSCs, and acetylation drives the opening of specific TADs ${ }^{32}$. The specific locations of these marks are, at least partially, preserved through the differentiation of HSCs into different myeloid and lymphoid lineage cells ${ }^{32}$. It is likely that in terminally differentiated myeloid cells, the $\mathrm{H} 3 \mathrm{~K} 4 \mathrm{mel}$ marks established in the HSC lineage continue to be present, and the gain and loss of $\mathrm{H} 3 \mathrm{~K} 27 \mathrm{ac}$ occurs in response to exposure to LPS tolerization and $\beta$-glucan priming, even though this question remains to be formally analysed and established. The transmission of these marks through DNA replication and the cell cycle is a central conundrum around how trained immunity is stably maintained ${ }^{111}$. Clearly, an as yet undiscovered mechanism preserves $\mathrm{H} 3 \mathrm{~K} 4 \mathrm{me} 1$ and $\mathrm{H} 3 \mathrm{~K} 4 \mathrm{me} 3$ marks through the cell cycle in HSCs.

In the adaptive responses of NK cells, epigenetic control of activation, clonal proliferation, contraction and memory have been demonstrated in the context of viral infection in both humans and mice ${ }^{112,113}$. Naive, effector and memory NK cells possess distinct chromatin accessibility states as determined by ATAC-seq and H3K4me3 chromatin immunoprecipitation followed by sequencing, which has revealed a 'poised' regulatory programme at the memory NK cell stage following MCMV infection $^{113}$. Furthermore, concurrent chromatin profiling of the MCMV-specific $\mathrm{CD}^{+} \mathrm{T}$ cell response demonstrated parallel epigenetic signatures that define memory NK cells and $\mathrm{CD}^{+} \mathrm{T}$ cells ${ }^{113}$. Finally, many transcription factors have been identified that promote permissive histone modifications and overall chromatin accessibility at specific loci to drive adaptive NK cell responses, including STAT4, STAT1, ZBTB32, T-bet, EOMES, IRF8, IRF9, KLF12 and RUNX family transcription factors $^{70,87,114-118}$. 


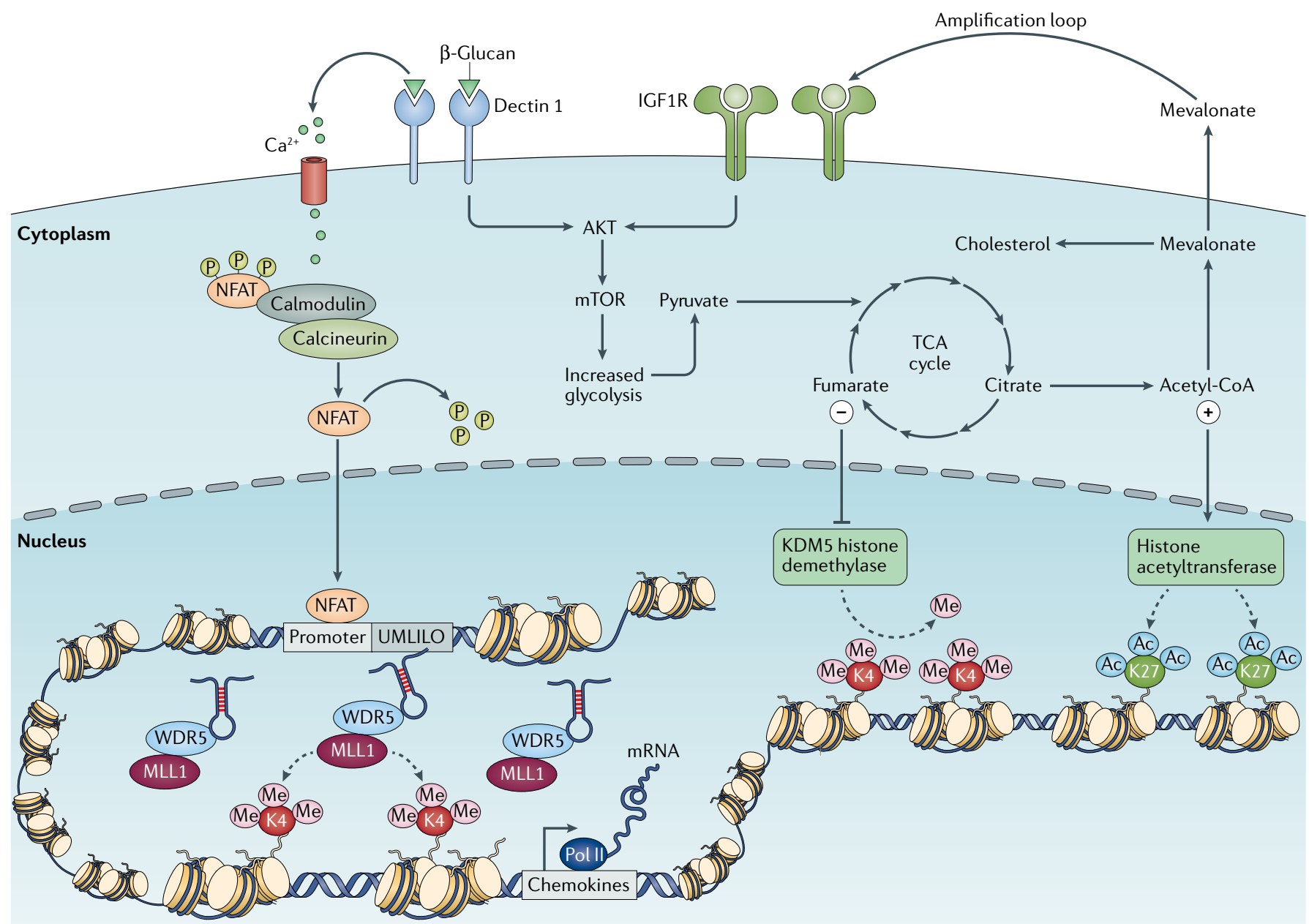

Fig. 3 | Interplay between epigenetics and metabolism. The correct initiation of the mechanisms necessary for the induction of trained immunity relies on the active interplay between epigenetic and metabolic reprogramming of the innate immune cells on stimulation. During primary challenge, the recognition of specific ligands by pattern recognition receptors triggers a series of intracellular cascades that lead to the upregulation of different metabolic pathways, such as glycolysis, tricarboxylic acid (TCA) cycle and fatty acid metabolism. Certain metabolites derived from these processes, such as fumarate and acetyl coenzyme A (acetyl-CoA), can activate or inhibit a series of enzymes involved in remodelling the epigenetic landscape of cells, such as the histone demethylase lysine-specific demethylase 5 (KDM5) or histone acetyltransferases, leading to specific changes in histone methylation and acetylation of genes involved in the innate immune responses. $\beta$-Glucan-mediated activation of dectin 1 signalling also triggers calcium influx, which leads to the dephosphorylation of nuclear factor of activated T cells (NFAT), allowing its translocation into the nucleus, where it may bind to DNA and activate gene transcription. This facilitates the accessibility of the DNA to the transcriptional machinery and gene regulatory elements and specific long non-coding RNAs, promoting and facilitating an enhanced gene transcription on secondary stimulation of the cells. IGF1R, insulin-like growth factor 1 receptor; MLL1, mixed-lineage leukaemia protein 1 (also known as histone-lysine $\mathrm{N}$-methyltransferase 2A); mTOR, mechanistic target of rapamycin; Pol, polymerase; UMLILO, upstream master long non-coding RNA of the inflammatory chemokine locus; WDR5, WD repeat-containing protein 5 .
MicroRNAs might also have a role in the induction and regulation of these mechanisms. miR-155 was shown to be critical for adaptive NK cell responses to MCMV infection through the regulation of targets, including NOXA and SOCS1 (REFS ${ }^{119,120}$ ). The upregulation of miR-155 during inflammatory processes has also been correlated with the hyperactivation of cells from the myeloid compartment. This is likely owing to a decreased activity of phosphatases that act as negative regulators of a series of intracellular pathways ${ }^{121}$, including the phosphatase SHIP1, which was recently demonstrated to act as a negative regulator in the induction of trained immunity ${ }^{122}$.

New studies also suggest that changes in DNA methylation patterns discriminate between 'responders' (people who are able to undergo trained immunity) and 'non-responders' to stimuli that induce trained immunity, such as BCG. In this regard, individuals who exhibit an enhanced containment of $M$. tuberculosis replication after BCG vaccination displayed a wide loss of DNA methylation among promoters of genes belonging to immune pathways compared with individuals characterized as non-responders ${ }^{123}$. A follow-up study identified 43 genes with differential methylation patterns in BCG-naive responders compared with non-responders that could potentially be used as predictors of responsiveness to stimuli that induce trained immunity ${ }^{124}$.

As mentioned earlier, non-haematopoietic cells, such as epidermal stem cells, also show features of trained immunity. The epigenetic memory of epidermal stem 


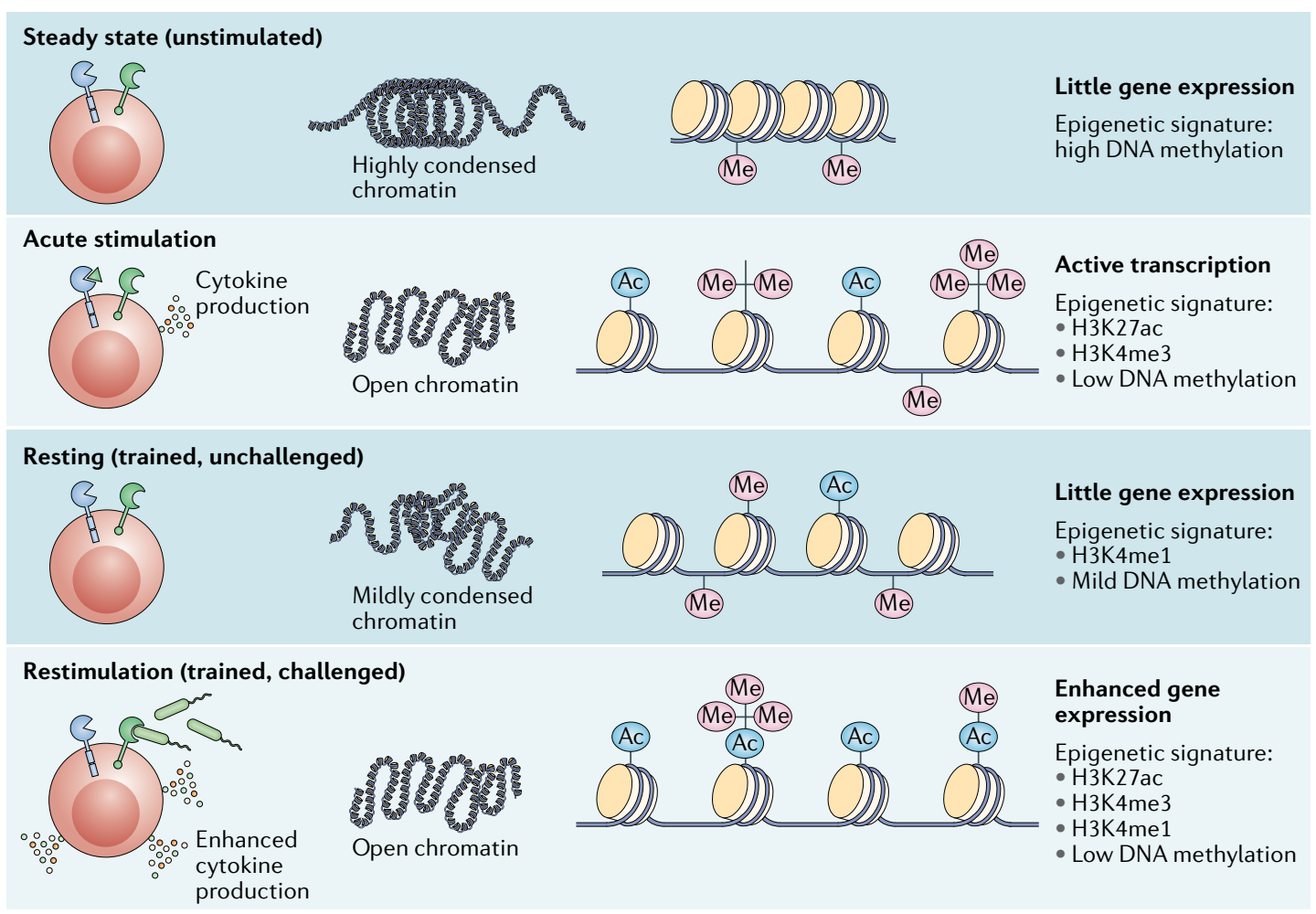

Fig. 4 | Epigenetic reprogramming underlies the induction of trained immunity. Stimulation of innate immune cells is accompanied by the deposition of chromatin marks and changes in the DNA methylation status, leading to unfolding of chromatin and facilitating transcription and expression of proinflammatory factors. All of these changes are only partially removed after cessation of the stimulus. This allows quicker and enhanced recruitment of transcription factors and gene expression after secondary challenge with another stimulus. The figure illustrates the chromatin states and epigenetic signatures associated with unstimulated cells, with cells following acute stimulation, with resting 'trained' cells and with trained cells following restimulation. $\mathrm{H} 3 \mathrm{~K} 27 \mathrm{ac}$, histone 3 lysine 27 acetylation; H3K4me, histone 3 lysine 4 methylation; H3K4me3, histone 3 lysine 4 trimethylation.

cells is also interesting as certain features of it can still be detected months after the inflammation has resolved ${ }^{6}$. Additionally, the lingering accessible chromatin domains harboured within the epidermal stem cells of inflammation-exposed skin can act as inflammation-sensing enhancers when excised and used to drive reporter expression in the skin in vivo. Thus, although the molecular mechanisms underlying this memory are still unfolding, as they are for inflammatory memories rooted in other cell types, the field is coming to the view that cells and tissues may have evolved to possess memory for the benefit of confronting recurrent disease pathologies and maintaining tissue fitness.

\section{Immunometabolic circuits}

Cellular metabolism is a critical mediator of the trained immunity-dependent epigenetic reprogramming of innate immune cells and their progenitors ${ }^{125-127}$ (FIG. 3). It is well established that metabolites can modulate the activity of chromatin-modifying enzymes ${ }^{128}$; hence, metabolic rewiring of innate immune cells or their progenitors will regulate their plasticity and epigenomic reprogramming in the context of trained immunity ${ }^{125}$. Increased aerobic glycolysis is a hallmark of $\beta$-glucan-trained monocytes; this is mediated by a pathway that involves the activity of AKT, mechanistic target of rapamycin (mTOR) and hypoxia-inducible factor $1 \alpha(\mathrm{HIF} 1 \alpha)$. Consistently, blockade of the AKTmTOR-HIF1 $\alpha$ pathway abrogates trained immunity ${ }^{129}$. Furthermore, BCG-induced trained immunity requires the functional reprogramming of monocyte metabolism towards aerobic glycolysis to develop enhanced responsiveness to subsequent stimulation ${ }^{130}$.

Subsequent studies with integrated metabolomic and transcriptomic analyses of human $\beta$-glucan-trained monocytes revealed crosstalk between glycolysis and glutaminolysis in trained immunity ${ }^{131}$. Trained monocytes accumulate the tricarboxylic acid cycle (TCA) metabolite fumarate, which influences epigenetic reprogramming by downregulating the activity of KDM5 histone demethylases ${ }^{131}$. Moreover, different TCA intermediates exert distinct effects on innate immune cell activity. For instance, $\alpha$-ketoglutarate promotes anti-inflammatory activation of macrophages via epigenetic reprogramming that is mediated by the $\mathrm{H} 3 \mathrm{~K} 27$ demethylase JMJD3. Moreover, $\alpha$-ketoglutarate facilitates endotoxin tolerance after classic LPS-mediated or IFN $\gamma$-mediated activation of macrophages ${ }^{132}$.

By contrast, LPS-induced succinate regulates a proinflammatory HIF $1 \alpha-I L-1 \beta$ pathway in mouse bone marrow-derived macrophages ${ }^{133}$. The metabolite itaconate, the concentration of which is highly upregulated in LPS-activated macrophages, exerts 
anti-inflammatory activity by inhibiting the succinate dehydrogenase-mediated oxidation of succinate to fumarate $\mathrm{e}^{134,135}$. Itaconate itself acts in an antiinflammatory fashion in macrophages by supporting the activity of the anti-inflammatory transcription factor NRF2 (REF. ${ }^{136}$ ) and the inhibition of LPS-mediated I $\kappa \mathrm{B}$ induction. The latter effect of itaconate is independent of NRF2 but requires activating transcription factor 3 (ATF3) ${ }^{137}$. Of note, itaconate-induced tolerance in human monocytes is counteracted by $\beta$-glucan-induced trained immunity as $\beta$-glucan inhibits the expression of immune-responsive gene 1 (IRG1) protein, the enzyme responsible for itaconate generation. Consistent with fumarate accumulation in $\beta$-glucan-trained monocytes $^{131}, \beta$-glucan-mediated inhibition of IRG1 results in elevated expression of succinate dehydrogenase ${ }^{138}$. Thus, $\beta$-glucan-induced trained immunity is associated with enhanced succinate dehydrogenase activity and accumulation of fumarate as well as with reversing the endotoxin tolerance-inducing effects of itaconate, which acts as an antagonist of succinate dehydrogenase.

Enhanced cholesterol synthesis is also an important hallmark of $\beta$-glucan-trained monocytes. The 3-hydroxy-3-methylglutaryl coenzyme A (HMG-CoA) reductase inhibitor fluvastatin blocks trained immunity in primary human monocytes ${ }^{131}$. Interestingly, this is accomplished not by cholesterol biosynthesis but rather by an accumulation of the upstream metabolite mevalonate. Brief exposure to mevalonate can trigger training of monocytes via the insulin-like growth factor 1 receptor and stimulation of mTOR signalling ${ }^{139}$. Importantly, enhanced cholesterol synthesis is critical for the $\beta$-glucan-induced training of not only mature myeloid cells but also of their progenitors (haematopoietic stem and progenitor cells (HSPCs)). The long-term myelopoiesis bias conferred to HSPCs by $\beta$-glucan-induced training is associated with accumulation of cholesterol esters and lipids with more saturated acyl chains ${ }^{104}$. Inhibition of HMG-CoA reductase diminishes $\beta$-glucan-induced HSPC population expansion and myelopoiesis ${ }^{104}$. The enhanced cholesterol levels that occur in HSPCs as a result of innate training may promote a myelopoiesis bias via upregulation of CD131, the common $\beta$-subunit of the IL-3/GM-CSF receptor ${ }^{104}$. This is consistent with findings showing that inhibition of cholesterol efflux in HSPCs, due to deficiency in the ATP-binding cassette transporter ABCA1, also enhances CD131 expression and myelopoiesis in the bone marrow $^{140,141}$.

\section{Pathological outcomes of trained immunity}

Infections were the most common causes of death throughout the world 150 years ago, and they continue to represent the most significant threats to health in low-income countries. Therefore, strong evolutionary pressure has shaped antimicrobial immune functions, including trained immunity. Although trained immunity evolved as a beneficial immune process to protect against infection, one may envisage situations in which reprogramming of innate immunity and increased inflammatory responses to exogenous or endogenous stimuli may also have harmful effects. It is becoming increasingly evident that sterile inflammation in response to lifestyle changes in Western societies forms the basis on which chronic inflammatory diseases develop ${ }^{142}$. It will thus be necessary to better understand how sterile inflammatory insults induce trained immunity and how trained immunity mechanisms could contribute to chronic inflammation in various diseases associated with Western lifestyle ${ }^{142}$.

Trained immunity and inflammatory diseases. It is possible that the augmented immune functions arising from trained immunity could lead to pathological tissue damage in certain situations. Trained immunity could, in part, explain the epidemiological link between infections and atherosclerotic cardiovascular disease ${ }^{143,144}$. In addition to microbial products, endogenous triggers of innate immunity, including oxidized low-density lipoprotein particles, lipoprotein $(a)$, vimentin and highmobility group box 1 (HMGB1), can induce trained immunity $^{145-147}$.

Recent work has assessed whether a Western-type diet (that is, a diet enriched in fats, sugars and salt and low in fibre) can induce trained immunity. In atherosclerosisprone $\mathrm{Ldlr}^{-1-}$ mice, 4 weeks of such a Western-type diet induced profound proinflammatory transcriptional and epigenetic reprogramming of circulating monocytes and their bone marrow myeloid progenitor cells. The dietary intervention induced increased inflammatory responses to subsequent innate immune stimuli. This trained immunity phenotype persisted even after the mice had been switched to a standard chow diet for another 4 weeks, despite circulating cholesterol levels and systemic inflammatory markers returning to nor$\mathrm{mal}^{8}$. Several small proof-of-principle studies in patients suggest that trained immunity also occurs in the setting of dyslipoproteinaemia: monocytes from patients with familial hypercholesterolaemia are characterized by an enhanced cytokine production capacity and enrichment of $\mathrm{H} 3 \mathrm{~K} 4 \mathrm{me} 3$ on their promoters, which remains present even after 3 months of cholesterol-lowering statin treatment ${ }^{148}$. Furthermore, circulating monocytes from patients with severe coronary artery atherosclerosis exhibit a trained immune phenotype in terms of enhanced cytokine production capacity and glycolytic metabolism and epigenetic reprogramming at the level of histone methylation ${ }^{149,150}$.

Another clinical scenario in which sterile endogenous stimuli could trigger trained immunity in monocyte-derived cells is organ transplantation ${ }^{146}$. Braza et al. ${ }^{146}$ recently showed in a mouse heart transplantation model that donor allografts upregulate vimentin and HMGB1, which induced local training of graft-infiltrating monocyte-derived cells. Short-term treatment with a high-density lipoprotein nanobiologic that specifically inhibited mTOR in myeloid cells was able to prevent aerobic glycolysis and epigenetic modifications underlying trained immunity. The resulting Ly6C ${ }^{\text {low }}$ monocyte-derived macrophage-like cells with a regulatory phenotype prevented alloreactive $\mathrm{CD}^{+}$ $\mathrm{T}$ cell-mediated immunity and promoted tolerogenic $\mathrm{CD}^{+}{ }^{+}$regulatory $\mathrm{T}$ cell expansion, which improved allograft survival. 
Trained immunity and neurodegenerative diseases. Trained immunity could potentially be important to ameliorate the consequences of immunosenescence, which is associated with the loss of adaptive immune system function. For example, prior BCG vaccination has been shown to enhance antibody responses to many other vaccines that are subsequently administered $^{151,152}$. On the other hand, there could be negative consequences. Neurodegenerative diseases constitute a significant group of age-related diseases associated with chronic inflammation ${ }^{153}$. Peripheral application of inflammatory stimuli in a mouse model of Alzheimer disease leads to long-lasting training of microglia, the brain-resident macrophages, which exacerbates cerebral $\beta$-amyloidosis ${ }^{154}$. The functional changes of microglia are accompanied by activating epigenetic changes at the HIF1A gene locus, consistent with the peripheral trained immunity response ${ }^{129}$. As a consequence of epigenetic reprogramming, microglia also show changes in transcription and protein expression. Even infections of mice very early in life as a means of immunological training seem to be able to contribute to the impairment of microglial function followed by amyloid- $\beta$ induced synapse damage and cognitive impairment ${ }^{155}$. Together, these studies suggest that systemic inflammation induces microglia reprogramming, resulting in potentially hyper-responsive 'trained' states of the brain immune system.

Another brain pathological abnormality linked to systemic inflammation and associated with dementia is cerebral small vessel disease ${ }^{156}$. In patients with cerebral small vessel disease, peripheral blood-derived monocytes showed trained immunity characteristics such as enhanced IL- 6 and IL- 8 production after ex vivo stimulation, which was also associated with the severity and progression of the disease ${ }^{157}$. A causal link to the pathophysiology of the small vessels in the brain remains to be determined. The pathophysiology of acute stroke is unaltered by prior peripheral immune stimulation ${ }^{154}$, which might suggest that chronic rather than acute inflammatory conditions are associated with both trained immunity and the induction of neuroinflammation and neurodegeneration. Accumulating evidence suggests that there is a link between trained immunity and 'inflammageing', the inflammatory condition related to an ageing immune system ${ }^{158}$. For example, age-related reprogramming of specific innate immune cells might enhance effector mechanisms associated with trained immunity (such as production of IL-8 (REF. ${ }^{159}$ ) and CCL1 (REF. $\left.{ }^{160}\right)$ ), thereby leading to hyper-reactivity. Collectively, a better understanding of the dark side of trained immunity in ageing populations might help us to fight inflammageing-related chronic diseases such as dementia in elderly patients.

Tumour growth and metastasis. Robust and efficient activation of the immune system is fundamental to eliminate cancer cells from the organism. However, excessive or prolonged inflammatory responses can also promote tumour progression as chronic inflammation fuels and sustains disease progression and neoplastic transformation in particular tumour entities. The induction of trained immunity and the metabolic processes in cancer cells share several common features, such as reliance on glycolytic metabolism and the upregulation of the expression and activity of transcription factors such as HIF1a. The induction of trained immunity can be either beneficial or detrimental in the interplay between tumour cells and the cells of the innate immune system.

On the other hand, innate immune cells infiltrating the microenvironment of specific tumours can undergo a reprogramming process that leads to the development of maintained inflammatory responses, so-called smouldering inflammation, that can increase the degree of antigen-driven lymphoproliferation ${ }^{161}$, impair apoptosis, promote mitochondrial dysfunction and increase oxidative stress in the tumour microenvironment, which ultimately promotes the progression of the tumour. Tumour cells can also reprogramme infiltrating innate immune cells to acquire a more anti-inflammatory phenotype that is reminiscent of the immunoparalysis observed in patients with sepsis; for instance, monocytes from patients with chronic lymphocytic leukaemia show low levels of cytokine production, high phagocytic activity and impaired antigen presentation ${ }^{162}$. Efficient long-term cell reprogramming is necessary to ensure the efficacy of pharmacological treatments directed against cancer, as shown by the failure of patients with cancer to develop durable responses after treatment with checkpoint inhibitors owing to epigenetic stability of exhausted T cells ${ }^{163}$. Cytokines such as IL-6 and tumour necrosis factor (TNF) that are produced by trained cells are associated with increased tumorigenicity and the spread of metastases in specific types of tumours, including oral squamous cell carcinoma and lung, kidney and breast cancer ${ }^{164,165}$. Cancer cells also produce a series of soluble mediators that can induce direct epigenetic and metabolic reprogramming in immune cells and can thereby contribute to the progression of the tumour ${ }^{166}$.

The data presented above suggest that the ability of immune cells to tune their responses to adapt to changing environments is an important feature that evolved to prepare immune cells for unpredictable events, such as pathogen invasion. However, the epigenetic mechanisms that control the memory of the environmental trigger may also lead to the persistence of pathological responses that drive disease.

\section{Conclusions and future challenges}

In this Review, we have presented evidence to suggest that trained immunity, as an epigenetic memory of inflammatory encounters, is a fundamental characteristic of host defence of multicellular organisms, including mammals.

There are many remaining questions and important lines of study that need to be followed in this exciting new field of immunology. One goal should be to describe in more detail the molecular mechanisms that mediate trained immunity. This should involve an exploration of the entire range of immune and non-immune cell populations in which trained immunity can be induced, as well as the precise definition of the immunological, metabolic and epigenetic processes that mediate trained immunity. We need to better understand how 


\section{Box 2 | Clinical relevance of inhibiting or reversing trained immunity}

Trained immunity is relevant to a range of conditions in which an exacerbated immune response drives disease progression, such as in inflammatory bowel disease, gout, allergy and atherosclerosis and its clinical consequences, namely myocardial infarction and stroke. Therefore, suppressing ongoing trained immunity or preventing its induction will be a relevant treatment modality for numerous diseases associated with a chronic inflammatory state. In addition, Ochando and colleagues ${ }^{146}$ recently implicated trained immunity in organ rejection. Using a trained immunity-inhibiting and mechanistic target of rapamycin (mTOR)-specific nanotherapeutic, they showed that a short treatment regimen resulted in prolonged allograft survival without the need for long-term immunosuppression in a mouse heart transplantation model. Notably, preventing trained immunity rebalanced the myeloid cell compartment from proinflammatory to anti-inflammatory, while the adaptive immune system was characterized by an increased frequency of regulatory $T$ cells. Co-treatment with a nanoimmunotherapeutic agent that prevents $\mathrm{CD} 40-\mathrm{CD} 40 \mathrm{~L}$ co-stimulation ${ }^{186}$ led to the induction of tolerance. These observations highlight that the innate and adaptive immune systems work in conjunction, and that immune memory can be best considered a feature relevant to phagocytes and lymphocytes ${ }^{187}$.

In addition to targeting myeloid cell metabolic pathways such as the mTOR or hypoxia-inducible factor 1 $\alpha$ pathway, inhibiting endogenous mediators of trained immunity such as the NLRP 3 inflammasome or IL- $1 \beta$ release is an alternative strategy to suppress trained immunity's inflammatory component. This was explored in patients with cardiovascular disease in the Canakinumab Anti-inflammatory Thrombosis Outcome Study (CANTOS) ${ }^{188}$. Finally, regulating epigenetic processes is another compelling therapeutic avenue towards inhibiting trained immunity. Suppression of trained immunity may also be achieved by restricting epigenetic changes with, for example, inhibitors of histone or DNA methylation. Moreover, after the promotion or inhibition of trained immunity, the duration of this phenomenon may be managed by epigenetic modulators that can maintain a certain state of the chromatin in relevant genomic regions. Small molecules that target various epigenetic enzymes (from histone deacetylase inhibitors to modulators of histone methylation and bromodomain inhibitors) are being developed for use in cancer therapy ${ }^{189,190}$. The applicability of such approaches for other immunomediated diseases is an important avenue to be investigated soon. Finally, approaches targeting such novel compounds to the relevant cell population, especially myeloid cells and their precursors, should be attempted for specific therapy with weak side effects. Bone marrow-targeted nanotherapeutics may offer such a path towards novel therapies.

long-lasting trained immunity is as a consequence of infections, vaccination or even sterile triggers, and the influence of the host microbiota on the trained immunity responses. A fascinating further area of research would be to investigate whether trained immunity can be transmitted epigenetically in the germline, as previously reported for plants and insects ${ }^{17,167-169}$.

An important area of future research will be to use the mechanisms induced by trained immunity for the design of a new generation of therapies and vaccines that combine induction of classical adaptive immune memory and trained immunity (BOX 2). The World Health Organization recommends more research into non-specific effects of vaccines ${ }^{170}$; so far, the evidence shows that a vaccination programme that ensures that live vaccines are given would provide optimal specific protection as well as trained immunity, and this would have a substantial impact on overall mortality ${ }^{171}$. Furthermore, new epidemiological study results intriguingly suggest that the non-specific beneficial effects of live vaccines may be amplified if the vaccines are given in the presence of pre-existing immunity, be it from parental priming or from a previous vaccine $\mathrm{e}^{16,172}$. Thus, a modified vaccination programme that provides live vaccines earlier, in the presence of maternal immunity, and in multiple doses may lead to increased innate training. Given that trained immunity can induce heightened immune responses, potential non-specific effects of vaccines with regard to chronic inflammatory conditions should be investigated.

Finally, one of the most critical future lines of research is to explore the impact of trained immunity on disease: how does trained immunity contribute to the pathogenesis of immune-mediated diseases on the one hand, and how can trained immunity be approached as a therapeutic target on the other hand? Trained immunity is expected to have an important role both in diseases with impaired host defence, such as postsepsis immune paralysis or cancers, and in autoinflammatory and autoimmune diseases, in which an exacerbated trained immunity phenotype could contribute to disease pathogenesis. The impact of trained immunity, and more generally of epigenetic rewiring in various processes of priming, adaptation or tolerance during disease ${ }^{111}$, warrants further studies. Also, the role of trained immunity during the ageing process, and the potential association with clonal haematopoiesis, is an important area for future research. On the basis of a profound understanding of these mechanisms, therapeutic applications of the concept of trained immunity are expected to emerge: new generations of vaccines that combine adaptive and innate immune memory ${ }^{173}$; development of inducers of trained immunity for the treatment of immune paralysis in cancer ${ }^{174,175}$ or sepsis ${ }^{13}$; and the modulation of the potentially deleterious consequences of trained immunity in immunomediated and neurodegenerative diseases. Only sustained efforts by the community of researchers working on trained immunity will be able to achieve these aims and fulfil the potential brought by the understanding of the role of trained immunity in health and disease.

Published online 4 March 2020
1. Medzhitov, R. $£$ Janeway, C. Innate immune recognition: mechanisms and pathways. Immunol. Rev. 173, 89-97 (2000).

2. Lanier, L. L. NK cell recognition. Annu. Rev. Immunol. 23, 225-274 (2005).

3. Bonilla, F. A. \& Oettgen, H. C. Adaptive immunity. J. Allergy Clin. Immunol. 125, S33-S40 (2010).

4. Bowdish, D. M. E., Loffredo, M. S., Mukhopadhyay, S. Mantovani, A. \& Gordon, S. Macrophage receptors implicated in the "adaptive" form of innate immunity. Microbes Infect. 9, 1680-1687 (2007).

5. Netea, M. G., Quintin, J. \& Van Der Meer, J. W. M. Trained immunity: a memory for innate host defense. Cell Host Microbe 9, 355-361 (2011).

This article describes the memory features of the innate immune system and proposes the term 'trained immunity' for the first time.
6. Naik, S. et al. Inflammatory memory sensitizes skin epithelial stem cells to tissue damage. Nature $\mathbf{5 5 0}$ 475-480 (2017).

This study shows that epithelial stem cells maintain chromosomal accessibility at key stress response genes that are activated by the primary stimulus

7. Lay, K. et al. Stem cells repurpose proliferation to contain a breach in their niche barrier. eLife 7 , e41661 (2018)

8. Christ, A. et al. Western diet triggers NLRP3-dependent innate immune reprogramming. Cell 172, 162-175 (2018).

This study found that a high-fat diet induces long-lasting functional reprogramming of immunity in myeloid cells that remains after mice are returned to a chow diet.
9. Kurtz, J. Specific memory within innate immune systems. Trends Immunol. 26, 186-192 (2005).

10. Conrath, U., Beckers, G. J. M., Langenbach, C. J. C. \& Jaskiewicz, M. R. Priming for enhanced defense. Annu. Rev. Phytopathol. 53, 97-119 (2015).

11. Gourbal, B. et al. Innate immune memory: an evolutionary perspective. Immunol. Rev. 283, 21-40 (2018).

12. Netea, M. G. et al. Trained immunity: a program of innate immune memory in health and disease. Science 352, aaf1098 (2016).

13. Novakovic, B. et al. $\beta$-glucan reverses the epigenetic state of Ips-induced immunological tolerance. Cell 167, 1354-1368.e14 (2016).

This study details the epigenetic and transcriptional landscape of human macrophage LPS-induced tolerance and characterizes the potential of $\beta$-glucan to reverse it. 
14. Nankabirwa, V. et al. Child survival and BCG vaccination: a community based prospective cohort study in Uganda. BMC Public Health 15, 175 (2015).

15. Dominguez-Andres, J. \& Netea, M. G. Long-term reprogramming of the innate immune system. J. Leukoc. Biol. 105, 329-338 (2018).

16. Berendsen, M. L. T. et al. Maternal priming: bacillus Calmette-Guérin (BCG) vaccine scarring in mothers enhances the survival of their child with a BCG vaccine scar. J. Pediatric Infect. Dis. Soc. https://doi.org/ 10.1093/jpids/piy 142 (2019).

17. Moore, R. S., Kaletsky, R. \& Murphy, C. T. Piwi/PRG-1 argonaute and TGF- $\beta$ mediate transgenerational learned pathogenic avoidance. Cell 177, 1827-1841 (2019).

18. Hirano, M., Das, S., Guo, P. \& Cooper, M. D. The evolution of adaptive immunity in vertebrates. in. Adv. Immunol. 109, 125-157 (2011).

19. Cooper, M. D. \& Alder, M. N. The evolution of adaptive immune systems. Cell 124, 815-822 (2006).

20. Purvis, A. $\&$ Hector, A. Getting the measure of biodiversity. Nature 405, 212-219 (2000).

21. Milutinović, B. \& Kurtz, J. Immune memory in invertebrates. Semin. Immunol. 28, 328-342 (2016).

22. Reimer-Michalski, E.-M. \& Conrath, U. Innate immune memory in plants. Semin. Immunol. 28, 319-327 (2016).

23. Kleinnijenhuis, J. et al. Bacille Calmette-Guerin induces NOD2-dependent nonspecific protection from reinfection via epigenetic reprogramming of monocytes. Proc. Natl Acad. Sci. USA 109, 17537-17542 (2012).

24. Di Luzio, N. R. \& Williams, D. L. Protective effect of glucan against systemic Staphylococcus aureus septicemia in normal and leukemic mice. Infect. Immun. 20, 804-810 (1978)

25. Marakalala, M. J. et al. Dectin-1 plays a redundant role in the immunomodulatory activities of $\beta$-glucan-rich ligands in vivo. Microbes Infect. 15, 511-515 (2013)

26. Krahenbuhl, J. L., Sharma, S. D., Ferraresi, R. W. \& Remington, J. S. Effects of muramyl dipeptide treatment on resistance to infection with Toxoplasma gondii in mice. Infect. Immun. 31, 716-722 (1981).

27. Ribes, S. et al. Intraperitoneal prophylaxis with $\mathrm{CpG}$ oligodeoxynucleotides protects neutropenic mice against intracerebral Escherichia coli $\mathrm{K} 1$ infection. J. Neuroinflammation 11, 14 (2014).

28. Muñoz, N. et al. Mucosal administration of flagellin protects mice from Streptococcus pneumoniae lung infection. Infect. Immun. 78, 4226-4233 (2010).

29. Zhang, B. et al. Viral infection. Prevention and cure of rotavirus infection via TLR5/NLRC4-mediated production of IL-22 and IL-18. Science 346, 861-865 (2014).

30. van't Wout, J. W., Poell, R. \& van Furth, R. The role of BCG/PPD-activated macrophages in resistance against systemic candidiasis in mice. Scand. J. Immunol. $\mathbf{3 6}$ 713-719 (1992)

31. Tribouley, J., Tribouley-Duret, J. \& Appriou, M. Effect of bacillus Callmette Guerin (BCG) on the receptivity of nude mice to Schistosoma mansoni. C. R. Seances Soc. Biol. Fil. 172, 902-904 (1978)

32. Kaufmann, E. et al. BCG educates hematopoietic stem cells to generate protective innate immunity against tuberculosis. Cell 172,176-190.e19 (2018). This article shows that access of BCG to the bone marrow changes the transcriptional landscape of HSCs and multipotent progenitors, leading to local cell expansion and enhanced myelopoiesis.

33. Bromuro, $\mathrm{C}$. et al. Interplay between protective and inhibitory antibodies dictates the outcome of experimentally disseminated candidiasis in recipients of a Candida albicans vaccine. Infect. Immun. 70, 5462-5470 (2002)

34. Polonelli, L. et al. Therapeutic activity of an engineered synthetic killer antiidiotypic antibody fragment against experimental mucosal and systemic candidiasis. Infect. Immun. 71, 6205-6212 (2003).

35. Bistoni, F. et al. Evidence for macrophage-mediated protection against lethal Candida albicans infection. Infect. Immun. 51, 668-674 (1986).

36. Bistoni, F. et al. Immunomodulation by a low-virulence, agerminative variant of Candida albicans. Further evidence for macrophage activation as one of the effector mechanisms of nonspecific anti-infectious protection. J. Med. Vet. Mycol. 26, 285-299 (1988).

37. Quintin, J. et al. Candida albicans infection affords protection against reinfection via functional reprogramming of monocytes. Cell Host Microbe 12 223-232 (2012)
38. Vecchiarelli, A. et al. Protective immunity induced by low-virulence Candida albicans: cytokine production in the development of the anti-infectious state. Cell. Immunol. 124, 334-344 (1989)

39. Tso, G. H. W. et al. Experimental evolution of a fungal pathogen into a gut symbiont. Science 362, 589-595 (2018).

40. Biering-Sørensen, S. et al. Early BCG-Denmark and neonatal mortality among infants weighing $<2500 \mathrm{~g}$ : a randomized controlled trial. Clin. Infect. Dis. 65 1183-1190 (2017).

41. Rieckmann, A. et al. Vaccinations against smallpox and tuberculosis are associated with better long-term survival: a Danish case-cohort study 1971-2010. Int. J. Epidemiol. 46, 695-705 (2017).

This study finds that vaccinia and BCG vaccinations are associated with greater long-term survival, which is not explained by the pathogen-specific immune protection that these vaccines offer.

42. Aaby, P. et al. Vaccinia scars associated with better survival for adults. An observational study from Guinea-Bissau. Vaccine 24, 5718-5725 (2006).

43. Aaby, P. et al. Randomized trial of BCG vaccination at birth to low-birth-weight children: beneficial nonspecific effects in the neonatal period? J. Infect. Dis. 204, 245-252 (2011)

44. Aaby, P. et al. Non-specific beneficial effect of measles immunisation: analysis of mortality studies from developing countries. BMJ 311, 481-485 (1995). This study reports that the standard-titre measles vaccine may confer a beneficial effect that is unrelated to specific protection against measles infection.

45. Aaby, P. et al. Non-specific effects of standard measles vaccine at 4.5 and 9 months of age on childhood mortality: randomised controlled trial. BMJ 341 , c6495 (2010).

46. Lund, N. et al. The effect of oral polio vaccine at birth on infant mortality: a randomized trial. Clin. Infect. Dis. 61, 1504-1511 (2015).

47. Andersen, A. et al. National immunization campaigns with oral polio vaccine reduce all-cause mortality: a natural experiment within seven randomized trials. Front. Public Health 6, 13 (2018)

48. Benn, C. S., Netea, M. G., Selin, L. K. \& Aaby, P. A small jab - a big effect: nonspecific immunomodulation by vaccines. Trends Immunol. 34, 431-439 (2013).

49. Kleinnijenhuis, J. et al. BCG-induced trained immunity in NK cells: role for non-specific protection to infection. Clin. Immunol. 155, 213-219 (2014).

50. Jensen, K. J. et al. Heterologous immunological effects of early BCG vaccination in low-birth-weight infants in Guinea-Bissau: a randomized-controlled trial. J. Infect. Dis. 211, 956-967 (2015).

51. Freyne, B. et al. Neonatal BCG vaccination influences cytokine responses to toll-like receptor ligands and heterologous antigens. J. Infect. Dis. 217, 1798-1808 (2018)

52. Aaby, P. et al. Differences in female-male mortality after high-titre measles vaccine and association with subsequent vaccination with diphtheria-tetanuspertussis and inactivated poliovirus: reanalysis of West African studies. Lancet 361, 2183-2188 (2003).

53. Blok, B. A. et al. Interacting non-specific immunological effects of BCG and TDAPF vaccinations: an explorative randomized trial. Clin. Infect. Dis. 70, 455-463 (2019).

54. Arts, R. J. W. et al. BCG vaccination protects against experimental viral infection in humans through the induction of cytokines associated with trained immunity. Cell Host Microbe 23, 89-100 (2018)

55. Walk, J. et al. Outcomes of controlled human malaria infection after BCG vaccination. Nat. Commun. 10 , 874 (2019).

56. McCall, M. B. B. et al. Plasmodium falciparum infection causes proinflammatory priming of human TLR responses. J. Immunol. 179, 162-171 (2007).

57. Ataide, M. A. et al. Malaria-induced NLRP12 NLRP3-dependent caspase-1 activation mediates inflammation and hypersensitivity to bacterial superinfection. PLoS Pathog. 10, e 1003885 (2014).

58. Fitzgerald, K. A. et al. Cutting edge: Plasmodium falciparum induces trained innate immunity. J. Immunol. 6, 1243-1248 (2018)

59. Redelman-Sidi, G., Glickman, M. S. \& Bochner, B. H. The mechanism of action of BCG therapy for bladder cancer-a current perspective. Nat. Rev. Urol. 11 153-162 (2014)

60. Stewart, J. H. \& Levine, E. A. Role of bacillus Calmette-Guérin in the treatment of advanced melanoma. Expert. Rev. Anticancer. Ther. 11, 1671-1676 (2011)
61. Powles, R. L. et al. Maintenance of remission in acute myelogenous leukaemia by a mixture of B.C.G. and irradiated leukaemia cells. Lancet 2, 1107-1110 (1977).

62. Villumsen, M. et al. Risk of lymphoma and leukaemia after bacille Calmette-Guérin and smallpox vaccination a Danish case-cohort study. Vaccine 27, 6950-6958 (2009).

63. Buffen, K. et al. Autophagy controls BCG-induced trained immunity and the response to intravesical BCG therapy for bladder cancer. PLoS Pathog. 10, e1004485 (2014)

64. Foster, S. L., Hargreaves, D. C. \& Medzhitov, R. Gene-specific control of inflammation by TLR-induced chromatin modifications. Nature 447, 972-978 (2007).

65. Saeed, S. et al. Epigenetic programming of monocyte-to-macrophage differentiation and trained innate immunity. Science 345, 1251086 (2014). This article shows that modification of the epigenetic landscape is behind the effects of trained immunity in human monocytes.

66. Chen, F. et al. Neutrophils prime a long-lived effector macrophage phenotype that mediates accelerated helminth expulsion. Nat. Immunol. 15, 938-946 (2014).

67. Hole, C. R. et al. Induction of memory-like dendritic cell responses in vivo. Nat. Commun. 10, 2955 (2019).

68. Barton, E. S. et al. Herpesvirus latency confers symbiotic protection from bacterial infection. Nature 447, 326-329 (2007).

69. Sun, J. C., Beilke, J. N. \& Lanier, L. L. Adaptive immune features of natural killer cells. Nature 457 557-561 (2009).

70. Sun, J. C. et al. Proinflammatory cytokine signaling required for the generation of natural killer cell memory. J. Exp. Med. 209, 947-954 (2012).

71. Björkström, N. K. et al. Rapid expansion and long-term persistence of elevated NK cell numbers in humans infected with hantavirus. J. Exp. Med. 208 13-21 (2011).

72. Della Chiesa, M. et al. Phenotypic and functional heterogeneity of human NK cells developing after umbilical cord blood transplantation: a role for human cytomegalovirus? Blood 119, 399-410 (2012).

73. Foley, B. et al. Human cytomegalovirus (CMV)-induced memory-like $\mathrm{NKG}_{2} \mathrm{C}^{+} \mathrm{NK}$ cells are transplantable and expand in vivo in response to recipient $\mathrm{CMV}$ antigen. J. Immunol. 189, 5082-5088 (2012).

74. Arase, H. Mocarski, E. S. Campbell, A. E., Hill, A. B. \& Lanier, L. L. Direct recognition of cytomegalovirus by activating and inhibitory NK cell receptors. Science 296, 1323-1326 (2002)

75. Brown, M. G. et al. Vital involvement of a natural killer cell activation receptor in resistance to viral infection. Science 292, 934-937 (2001).

76. Adams, N. M. et al. Cytomegalovirus infection drives avidity selection of natural killer cells. Immunity $\mathbf{5 0}$, 1381-1390.e5 (2019).

77. Grassmann, S. et al. Distinct surface expression of activating receptor Ly $49 \mathrm{H}$ drives differential expansion of NK cell clones upon murine cytomegalovirus infection. Immunity 50, 1391-1400. e4 (2019).

78. Sun, J. C. \& Lanier, L. L. NK cell development, homeostasis and function: parallels with $\mathrm{CD} 8{ }^{+} \mathrm{T}$ cells. Nat. Rev. Immunol. 11, 645-657 (2011).

79. Min-Oo, G. \& Lanier, L. L. Cytomegalovirus generates long-lived antigen-specific NK cells with diminished bystander activation to heterologous infection. J. Exp. Med. 211, 2669-2680 (2014).

80. Rölle, A. et al. IL-12-producing monocytes and HLA-E control HCMV-driven NKG2 $\mathrm{C}^{+} \mathrm{NK}$ cell expansion. J. Clin. Invest. 124, 5305-5316 (2014).

81. Hammer, Q. et al. Peptide-specific recognition of human cytomegalovirus strains controls adaptive natural killer cells. Nat. Immunol. 19, 453-463 (2018).

82. Karo, J. M., Schatz, D. G. \& Sun, J. C. The RAC recombinase dictates functional heterogeneity and cellular fitness in natural killer cells. Cell 159, 94-107 (2014).

83. Vivier, E. et al. Innate or adaptive immunity? The example of natural killer cells. Science 331, 44-49 (2011).

84. Cooper, M. A. et al. Cytokine-induced memory-like natural killer cells. Proc. Natl Acad. Sci. USA 106, 1915-1919 (2009).

85. Romee, R. et al. Cytokine activation induces human memory-like NK cells. Blood 120, 4751-4760 (2012). 
86. Romee, R. et al. Cytokine-induced memory-like natural killer cells exhibit enhanced responses against myeloid leukemia. Sci. Transl Med. 8, 357ra1 23 (2016).

87. Madera, S. et al. Type I IFN promotes NK cell expansion during viral infection by protecting NK cells against fratricide. J. Exp. Med. 213, 225-233 (2016)

88. Madera, S. \& Sun, J. C. Cutting edge: stage-specific requirement of IL-18 for antiviral NK cell expansion. J. Immunol. 194, 1408-1412 (2015)

89. Nabekura, T., Girard, J.-P. \& Lanier, L. L. IL-33 receptor ST2 amplifies the expansion of NK cells and enhances host defense during mouse cytomegalovirus infection. J. Immunol. 194, 5948-5952 (2015).

90. Weizman, O.-E. et al. Mouse cytomegalovirusexperienced ILC $1 \mathrm{~s}$ acquire a memory response dependent on the viral glycoprotein $\mathrm{m} 12$ Nat. Immunol. 20, 1004-1011 (2019).

91. Martinez-Gonzalez, I. et al. Allergen-experienced group 2 innate lymphoid cells acquire memory-like properties and enhance allergic lung inflammation. Immunity 45, 198-208 (2016)

92. Paust, $\mathrm{S}$ et al. Critical role for the chemokine receptor CXCR6 in NK cell-mediated antigen-specific memory of haptens and viruses. Nat. Immunol. 11, 1127-1135 (2010)

93. Gamliel, M. et al. Trained memory of human uterine NK cells enhances their function in subsequent pregnancies. Immunity 48, 951-962 (2018). This study finds that uterine NK cells adapt to pregnancy and can better support subsequent pregnancies.

94. Gonzales, K. A. U. \& Fuchs, E. Skin and its regenerative powers: an alliance between stem cells and their niche. Dev. Cell 43, 387-401 (2017).

95. Naik, S., Larsen, S. B., Cowley, C. J. \& Fuchs, E. Two to tango: dialog between immunity and stem cells in health and disease. Cell 175, 908-920 (2018).

96. Biton, M. et al. T helper cell cytokines modulate intestinal stem cell renewal and differentiation. Cell 175, 1307-1320 (2018)

97. Lindemans, C. A. et al. Interleukin-22 promotes intestinal-stem-cell-mediated epithelial regeneration. Nature 528, 560-564 (2015)

98. von Moltke, J., Ji, M., Liang, H.-E. \& Locksley, R. M. Tuft-cell-derived IL-25 regulates an intestinal ILC2epithelial response circuit. Nature 529, 221-225 (2016).

99. Kamada, R. et al. Interferon stimulation creates chromatin marks and establishes transcriptional memory. Proc. Natl Acad. Sci. USA 115, E9162-E9171 (2018)

100. Merad, M., Sathe, P., Helft, J., Miller, J. \& Mortha, A The dendritic cell lineage: ontogeny and function of dendritic cells and their subsets in the steady state and the inflamed setting. Annu. Rev. Immunol. 31 563-604 (2013)

101. Yona, S. et al. Fate mapping reveals origins and dynamics of monocytes and tissue macrophages under homeostasis. Immunity 38, 79-91 (2013).

102. Patel, A. A. et al. The fate and lifespan of human monocyte subsets in steady state and systemic inflammation. J. Exp. Med. 214, 1913-1923 (2017).

103. Chavakis, T., Mitroulis, I. \& Hajishengallis, C Hematopoietic progenitor cells as integrative hubs for adaptation to and fine-tuning of inflammation. Nat. Immunol. 20, 802-811 (2019).

104. Mitroulis, I. et al. Modulation of myelopoiesis progenitors is an integral component of trained immunity. Cell 172, 147-161 (2018). This study shows that the induction of trained immunity modulates metabolism of haematopoietic progenitors in the bone marrow and increases myelopoiesis.

105. Ordovas-Montanes, J. et al. Allergic inflammatory memory in human respiratory epithelial progenitor cells. Nature 560, 649-654 (2018).

This article shows that epithelial stem cells contribute to the persistence of human disease by serving as repositories for allergic memories.

106. Machiels, B. et al. A gammaherpesvirus provides protection against allergic asthma by inducing the replacement of resident alveolar macrophages with regulatory monocytes. Nat. Immunol. 18, 1310-1320 (2017).

107. Yao, Y. et al. Induction of autonomous memory alveolar macrophages requires $\mathrm{T}$ cell help and is critical to trained immunity. Cell 175, 1634-1650. e17 (2018).

This study finds that the formation and maintenance of alveolar macrophages showing memory features occurs independently of monocytes or bone marrow progenitors.
108. Smale, S. T., Tarakhovsky, A. \& Natoli, G. Chromatin contributions to the regulation of innate immunity. Annu. Rev. Immunol. 32, 489-511 (2014).

109. Ghisletti, S. et al. Identification and characterization of enhancers controlling the inflammatory gene expression program in macrophages. Immunity 32 . 317-328 (2010)

110. Fanucchi, S. et al. Immune genes are primed for robust transcription by proximal long noncoding RNAs located in nuclear compartments. Nat. Genet. $\mathbf{5 1}$ 138-150 (2019).

This study hows that IncRNA-mediated regulation is central to the establishment of trained immunity

111. Natoli, G. \& Ostuni, R. Adaptation and memory in immune responses. Nat. Immunol. 20, 783-792 (2019).

112. Luetke-Eversloh, M. et al. Human cytomegalovirus drives epigenetic imprinting of the IFNG locus in NKG2Chi natural killer cells. PLoS Pathog. 10, e1004441 (2014).

113. Lau, C. M. et al. Epigenetic control of innate and adaptive immune memory. Nat. Immunol. 19 963-972 (2018)

114. Rapp, M. et al. Core-binding factor $\beta$ and Runx transcription factors promote adaptive natural killer cell responses. Sci. Immunol. 2, eaan3796 (2017).

115. Geary, C. D. et al. Non-redundant ISGF3 components promote NK cell survival in an auto-regulatory manner during viral infection. Cell Rep. 24, 1949-1957.e6 (2018).

116. Lam, V. C., Folkersen, L., Aguilar, O. A. \& Lanier, L. L. KLF1 2 regulates mouse NK cell proliferation. J. Immunol. 203, 981-989 (2019).

117. Adams, N. M. et al. Transcription factor IRF8 orchestrates the adaptive natural killer cell response. Immunity 48, 1172-1182.e6 (2018).

118. Madera, S. et al. Cutting edge: divergent requirement of T-box transcription factors in effector and memory NK cells. J. Immunol. 200, 1977-1981 (2018)

119. Zawislak, C. L. et al. Stage-specific regulation of natural killer cell homeostasis and response against viral infection by microRNA-155. Proc. Natl Acad. Sci. USA 110, 6967-6972 (2013).

120. Lu, L.-F. et al. A single miRNA-mRNA interaction affects the immune response in a context- and cell-type-specific manner. Immunity 43, 52-64 (2015)

121. O'Connell, R. M., Chaudhuri, A. A., Rao, D. S $\&$ Baltimore, D. Inositol phosphatase SHIP1 is a primary target of miR-155. Proc. Natl Acad. Sci. USA 106, 7113-7118 (2009).

122. Saz-Leal, P. et al. Targeting SHIP-1 in myeloid cells enhances trained immunity and boosts response to infection. Cell Rep. 25, 1118-1126 (2018).

123. Verma, D. et al. Anti-mycobacterial activity correlates with altered DNA methylation pattern in immune cells from BCG-vaccinated subjects. Sci. Rep. 7, 12305 (2017).

124. Das, J., Verma, D Gustafsson, M. \& Lerm, M. Identification of DNA methylation patterns predisposing for an efficient response to $\mathrm{BCC}$ vaccination in healthy BCG-naïve subjects. Epigenetics 14, 589-601 (2019).

125. Penkov, S., Mitroulis, I., Hajishengallis, G. \& Chavakis, T. Immunometabolic crosstalk: an ancestral principle of trained immunity? Trends Immunol. 40, 1-11 (2019).

126. Norata, G. D. et al. The cellular and molecular basis of translational immunometabolism. Immunity $\mathbf{4 3}$, 421-434 (2015).

127. Domínguez-Andrés, J., Joosten, L. A. \& Netea, M. G. Induction of innate immune memory: the role of cellular metabolism. Curr. Opin. Immunol. 56, 10-16 (2019).

128. Donohoe, D. R. \& Bultman, S. J. Metaboloepigenetics: interrelationships between energy metabolism and epigenetic control of gene expression. J. Cell. Physiol. 227, 3169-3177 (2012)

129. Cheng, S.-C. et al. mTOR- and HIF-1-mediated aerobic glycolysis as metabolic basis for trained immunity. Science 345, 1250684-1250684 (2014). This study reports that metabolic rewiring of cells is crucial for the induction of trained immunity.

130. Arts, R. J. W. et al. Immunometabolic pathways in BCG-induced trained immunity. Cell Rep. 17 2562-2571 (2016)

131. Arts, R. J. W. et al. Glutaminolysis and fumarate accumulation integrate immunometabolic and epigenetic programs in trained immunity. Cell Metab. https://doi.org/10.1016/j.cmet.2016.10.008 (2016).

132. Liu, P.-S. et al. a-Ketoglutarate orchestrates macrophage activation through metabolic and epigenetic reprogramming. Nat. Immunol. 18, 985-994 (2017)
133. Tannahill, G. M. et al. Succinate is an inflammatory signal that induces IL-1 $\beta$ through HIF-1 $\alpha$. Nature 496 238-242 (2013)

134. Lampropoulou, V. et al. Itaconate links inhibition of succinate dehydrogenase with macrophage metabolic remodeling and regulation of inflammation Cell Metab. 24, 158-166 (2016).

135. Cordes, T. et al. Immunoresponsive gene 1 and itaconate inhibit succinate dehydrogenase to modulate intracellular succinate levels. J. Biol. Chem. 291, 14274-14284 (2016).

136. Mills, E. L. et al. Itaconate is an anti-inflammatory metabolite that activates Nrf2 via alkylation of KEAP1. Nature 556, 113-117 (2018).

137. Bambouskova, M. et al. Electrophilic properties of itaconate and derivatives regulate the $\mathrm{I}_{\kappa} \mathrm{B} \zeta-\mathrm{ATF} 3$ inflammatory axis. Nature 556, 501-504 (2018).

138. Dominguez-Andres, J. et al. The itaconate pathway is a central regulatory node linking innate immune tolerance and trained immunity. Cell Metab. 29, 211-220 (2018).

139. Bekkering, S. et al. Metabolic induction of trained immunity through the mevalonate pathway. Cell 172 135-146.e9 (2018).

140. Yvan-Charvet, L. et al. ATP-binding cassette transporters and HDL suppress hematopoietic stem cell proliferation. Science 328, 1689-1693 (2010)

141. Murphy, A. J. et al. ApoE regulates hematopoietic stem cell proliferation, monocytosis, and monocyte accumulation in atherosclerotic lesions in mice. J. Clin. Invest. 121, 4138-4149 (2011).

142. Christ, A. \& Latz, E. The Western lifestyle has lasting effects on metaflammation. Nat. Rev. Immunol. 19, 267-268 (2019)

143. Musher, D. M., Abers, M. S. \& Corrales-Medina, V. F. Acute infection and myocardial infarction. N. Engl. J. Med. 380, 171-176 (2019).

144. Leentjens, J. et al. Trained innate immunity as a novel mechanism linking infection and the development of atherosclerosis. Circ. Res. 122, 664-669 (2018).

145. Bekkering, S. et al. Oxidized low-density lipoprotein induces long-term proinflammatory cytokine production and foam cell formation via epigenetic reprogramming of monocytes. Arterioscler. Thromb. Vasc. Biol. 34, 1731-1738 (2014)

146. Braza, M. S. et al. Inhibiting inflammation with myeloid cell-specific nanobiologics promotes organ transplant acceptance. Immunity 49, 819-828 (2018).

147. van der Valk, F. M. et al. Oxidized phospholipids on lipoprotein(a) elicit arterial wall inflammation and an inflammatory monocyte response in humans. Circulation 134, 611-624 (2016).

148. Bekkering, S. et al. Treatment with statins does not revert trained immunity in patients with familial hypercholesterolemia. Cell Metab. 30, 1-2 (2019).

149. Bekkering, S. et al. Innate immune cell activation and epigenetic remodeling in symptomatic and asymptomatic atherosclerosis in humans in vivo. Atherosclerosis 254, 228-236 (2016).

150. Shirai, T. et al. The glycolytic enzyme PKM2 bridges metabolic and inflammatory dysfunction in coronary artery disease J. Exp. Med 213,337-354 (2016).

151. Zimmermann, P., Finn, A. \& Curtis, N. Does BCG vaccination protect against nontuberculous mycobacterial infection? A systematic review and meta-analysis. J. Infect. Dis. 218, 679-687 (2018).

152. Leentjens, J et al. BCG vaccination enhances the immunogenicity of subsequent influenza vaccination in healthy volunteers: a randomized, placebo-controlled pilot study. J. Infect. Dis. 212, 1930-1938 (2015).

153. Ransohoff, R. M. How neuroinflammation contributes to neurodegeneration. Science 353, 777-783 (2016).

154. Wendeln, A.-C. et al. Innate immune memory in the brain shapes neurological disease hallmarks. Nature 556, 332-338 (2018)

155. Frost, P. S. et al. Neonatal infection leads to increased susceptibility to $A \beta$ oligomer-induced brain inflammation, synapse loss and cognitive impairment in mice. Cell Death Dis. 10, 323 (2019).

156. Low, A., Mak, E., Rowe, J. B., Markus, H. S \& O'Brien, J. T. Inflammation and cerebral small vessel disease: a systematic review. Ageing Res. Rev. 53, 100916 (2019).

157. Noz, M. P. et al. Trained immunity characteristics are associated with progressive cerebral small vessel disease. Stroke 49, 2910-2917 (2018)

158. Franceschi, C. et al. Immunobiography and the heterogeneity of immune responses in the elderly: a focus on inflammaging and trained immunity. Front. Immunol. 8, 982 (2017). 
159. Qian, F. et al. Age-associated elevation in TLR5 leads to increased inflammatory responses in the elderly. Aging Cell 11, 104-110 (2012).

160. Metcalf, T. U. et al. Global analyses revealed age-related alterations in innate immune responses after stimulation of pathogen recognition receptors. Aging Cell 14, 421-432 (2015).

161. Hoogeboom, R. et al. A mutated B cell chronic lymphocytic leukemia subset that recognizes and responds to fungi. J. Exp. Med. 210, 59-70 (2013).

162. Jurado-Camino, T. et al. Chronic lymphocytic leukemia: a paradigm of innate immune cross-tolerance. J. Immunol. 194, 719-727 (2015).

163. Pauken, K. E. et al. Epigenetic stability of exhausted $\mathrm{T}$ cells limits durability of reinvigoration by PD-1 blockade. Science 354, 1160-1165 (2016).

164. Lee, S. H. et al. TNF $\alpha$ enhances cancer stem cell-like phenotype via Notch-Hes1 activation in oral squamous cell carcinoma cells. Biochem. Biophys. Res. Commun. 424, 58-64 (2012)

165. Hodge, D. R., Hurt, E. M. \& Farrar, W. L. The role of IL- 6 and STAT3 in inflammation and cancer. Eur. J. Cancer 41, 2502-2512 (2005).

166. Rabold, K., Netea, M. G., Adema, G. J. \& Netea-Maier, R. T. Cellular metabolism of tumor-associated macrophages - functional impact and consequences. FEBS Lett. 591, 3022-3041 (2017).

167. Cubas, P., Vincent, C. \& Coen, E. An epigenetic mutation responsible for natural variation in floral symmetry. Nature 401, 157-161 (1999).

168. Greer, E. L. et al. A histone methylation network regulates transgenerational epigenetic memory in C. elegans. Cell Rep. 7, 113-126 (2014).

169. Tate, A. T., Andolfatto, P., Demuth, J. P. \& Graham, A. L. The within-host dynamics of infection in transgenerationally primed flour beetles. Mol. Ecol. 26, 3794-3807 (2017)

170. No Authors Listed. Meeting of the Strategic Advisory Group of Experts on immunization, April 2014 conclusions and recommendations. Wkly Epidemiol. Rec. 89, 221-236 (2014).

171. Shann, F. The heterologous (non-specific) effects of vaccines: implications for policy in high-mortality countries. Trans. R. Soc. Trop. Med. Hyg. 109, 5-8 (2015).

172. Benn, C. S., Fisker, A. B., Whittle, H. C. \& Aaby, P. Revaccination with live attenuated vaccines confer additional beneficial nonspecific effects on overall survival: a review. EBioMedicine 10, 312-317 (2016).

173. Locht, C. \& Mielcarek, N. Live attenuated vaccines against pertussis. Expert. Rev. Vaccines 13, 1147-1158 (2014)
174. Meyers, P. A. et al. Osteosarcoma: the addition of muramyl tripeptide to chemotherapy improves overall survival-a report from the Children's Oncology Group. J. Clin. Oncol. 26, 633-638 (2008).

175. Muramatsu, D. et al. $\beta$-glucan derived from Aureobasidium pullulans is effective for the prevention of influenza in mice. PLoS One 7, e41399 (2012).

176. Mulder, W. J. M., Ochando, J., Joosten, L. A. B., Fayad, Z. A. \& Netea, M. G. Therapeutic targeting of trained immunity. Nat. Rev. Drug. Discov. 18, 553-566 (2019).

177. Levine, D. B. The Hospital for the Ruptured and Crippled: William Bradley Coley, third Surgeon-in-Chief 1925-1933. HSS J. 4, 1-9 (2008).

178. Coley, W. B. II. Contribution to the knowledge of sarcoma. Ann. Surg. 14, 199-220 (1891).

179. Old, L. J., Clarke, D. A. \& Benacerraf, B. Effect of bacillus Calmette-Guérin infection on transplanted tumours in the mouse. Nature 184, 291-292 (1959).

180. Ostadrahimi, A. et al. Effect of beta glucan on white blood cell counts and serum levels of IL- 4 and IL- 12 in women with breast cancer undergoing chemotherapy: a randomized double-blind placebo-controlled clinical trial. Asian Pac. J. Cancer Prev. 15, 5733-5739 (2014).

181. Bashir, K. M. I. \& Choi, J.-S. Clinical and physiological perspectives of $\beta$-glucans: the past, present, and future. Int. J. Mol. Sci. 18, 1906 (2017)

182. Vetvicka, V., Vannucci, L., Sima, P. \& Richter, J. Beta glucan: supplement or drug? from laboratory to clinical trials. Molecules 24, 1251 (2019).

183. Lim, W. A. \& June, C. H. The principles of engineering immune cells to treat. Cancer Cell 168, 724-740 (2017).

184. Sharma, P. \& Allison, J. P. The future of immune checkpoint therapy. Science 348, 56-61 (2015).

185. Routy, B. et al. The gut microbiota influences anticancer immunosurveillance and general health. Nat. Rev. Clin. Oncol. 15, 382-396 (2018).

186. Lameijer, M. et al. Efficacy and safety assessment of a TRAF6-targeted nanoimmunotherapy in atherosclerotic mice and non-human primates. Nat. Biomed. Eng. 2 , 279-292 (2018)

187. Netea, M. G., Schlitzer, A., Placek, K., Joosten, L. A. B. $\&$ Schultze, J. L. Innate and adaptive immune memory: an evolutionary continuum in the host's response to pathogens. Cell Host Microbe 25, 13-26 (2019).

188. Ridker, P. M. et al. Antiinflammatory therapy with canakinumab for atherosclerotic disease. N. Engl. J. Med. 377, 1119-1131 (2017).

189. Domínguez-Andrês, J. et al. Bromodomain inhibitor I-BET151 suppresses immune responses during fungal-immune interaction. Eur. J. Immunol. 49, 2044-2050 (2019).
190. Alqahtani, A. et al. Bromodomain and extra-terminal motif inhibitors: a review of preclinical and clinical advances in cancer therapy. Future Sci. OA 5, FSO372 (2019).

\section{Acknowledgements}

M.G.N. was supported by a Spinoza grant from the Netherlands Organization for Scientific Research and a European Research Council (ERC) Advanced Grant (no. 833247). E.F. is an Investigator of the Howard Hughes Medical Institute and is supported by grants from the US National Institutes of Health (R01-AR31737 and R01-AR050452) and New York State (C32585GG). J.C.S. is supported by the Ludwig Center for Cancer Immunotherapy, the American Cancer Society, the Burroughs Wellcome Fund and the US National Institutes of Health (Al100874, Al130043 and P30CA008748). E.L. is supported by grants from the Deutsche Forschungsgemeinschaft (SFBs 645, 670 and 1123; TRRs 83 and 57), a grant from the US National Institutes of Health (1R01 HL11 2661) and an ERC Consolidator grant (InflammAct). J.L.S is supported by grants from the Deutsche Forschungsgemeinschaft (West German Cenome Center grant Central Coordination Unit of the national Next-Generation Sequencing Competence Network) and grants from the Helmholtz Gemeinschaft (Sparse2Big and AmPro) and the European Union (SYSCID, no. 733100). E.L., M.G.N., J.L.S and A.S are members of the Immuno-Sensation excellence cluster funded by the Deutsche Forschungsgemeinschaft under Germany's Excellence Strategy (EXC2151 390873048). T.C. is supported by an ERC Consolidator grant (DEMETINL) and by grants from the Deutsche Forschungsgemeinschaft (SFB 1181, TRR-SFB 205 and TRR-SFB 127). N.P.R., L.A.B.J. and M.G.N. received funding from the European Union Horizon 2020 research and innovation programme under grant agreement no. 667837 and the IN-CONTROL grant from the Dutch Foundation Netherlands (CVON2012-03 and CVON2018-27). N.P.R. is a recipient of a grant from European Research Area Network on Cardiovascular Diseases Joint Transnational Call 2018, which is supported by the Dutch Heart Foundation (JTC2018, project MEMORY; 2018T093).

Author contributions

The authors contributed equally to all aspects of the article.

Competing interests

The authors declare no competing interests.

Peer review information

Nature Reviews Immunology thanks H. Goodridge and the other, anonymous, reviewer(s) for their contribution to the peer review of this work.

\section{Publisher's note}

Springer Nature remains neutral with regard to jurisdictional claims in published maps and institutional affiliations.

(c) Springer Nature Limited 2020 